\title{
Consistent analysis of fusion data without adjustable parameters for a wide variety of heavy-ion systems
}

\author{
G. P. A. Nobre and L. C. Chamon \\ Departamento de Física Nuclear, Instituto de Física da Universidade de São Paulo, \\ Caixa Postal 66318, 05315-970, São Paulo, SP, Brazil \\ L. R. Gasques \\ Department of Nuclear Physics, Research School of Physical Sciences and Engineering, \\ Australian National University, Canberra ACT 0200, Australia \\ B. V. Carlson \\ Departamento de Física, Instituto Tecnológico de Aeronáutica, Centro Técnico Aeroespacial, \\ São José dos Campos, SP, Brazil \\ I. J. Thompson \\ University of Surrey, Guildford, Surrey GU2 7XH, United Kingdom \\ (Received 25 September 2006; published 19 April 2007)
}

\begin{abstract}
We have calculated fusion cross sections for 64 heavy-ion systems based on a method that takes into account the couplings to a complete set of states for surface vibrations of the nuclear densities. For the nuclear interaction we have assumed the parameter-free São Paulo potential. The predictions have been obtained without using any adjustable parameter, and are in good agreement with the experimental results for most of the systems, even at sub-barrier energies.
\end{abstract}

DOI: 10.1103/PhysRevC.75.044606

PACS number(s): 25.60.Pj, 25.70.Jj

\section{INTRODUCTION}

The heavy-ion fusion process has been extensively studied over the last decade [1], and a large amount of data has already been obtained for a wide variety of systems. Even so, many questions in this field are not completely solved yet. It is well known that fusion cross section data for heavy-ion systems show large enhancements at sub-barrier energies in comparison with theoretical predictions from the unidimensional barrier penetration model (BPM) [2]. In many works (e.g., [3,4]), the enhancements have been explained for several particular systems by considering the internal structure of the participating nuclei through coupled-channel (CC) calculations. CC data analyses and the realization of the connection between energy derivatives of the cross section and barrier distributions [5] have motivated the obtainment of high precision data. In recent works (see, e.g., [6-10]) the heavy-ion fusion at extreme sub-barrier energies is discussed. In some of these works, it has been observed that the diffuseness values for potentials adjusted to reproduce fusion data are significantly greater than those usually assumed for elastic scattering data analyses. Therefore, the consistency of the fusion and the elastic scattering data analyses is a goal to be reached.

Although from a theoretical point of view the $\mathrm{CC}$ formalism is appropriated to describe the heavy-ion fusion, numerical problems may occur in the solution of the $\mathrm{CC}$ equations resulting in unreliable theoretical cross sections, mainly at extreme sub-barrier energies, when a large number of channels is included in the calculations. This is an important subject since, for instance, recent data analyses [11] have demonstrated the sensitivity to multiphonon excitations in heavy-ion fusion reactions. On the other hand, several attempts to improve the BPM have been performed by considering permanent and/or vibrational deformations of the nuclei (e.g., [12]). For inelastic excitations, within the frozen approximation (FA) the transmission coefficient can be obtained by performing an average over different configurations of the system. For negligible excitation energies, this procedure is equivalent to full CC calculations where the complete basis of states is considered. The effect of finite excitation energy has already been studied in several works (see, e.g., [13-16]). In the present paper, we generalize the results obtained in Ref. [14] with the aim of performing calculations of heavy-ion fusion. Within a model that we call as zero point motion (ZPM), we consider the effect of the couplings to the complete sets of inelastic states connected to the $2^{+}$and $3^{-}$vibrational bands of even-even nuclei, taking into account the effect of the excitation energy. Despite the large number of coupled channels, the numerical convergence of our calculations is quite good.

In most works that deal with fusion data analyses, a few adjustable parameters related to the bare potential and/or coupling amplitudes have been used in order to fit the data. Despite the good data description generally obtained, this procedure may result in unrealistic values for the free parameters that could hide some particular characteristic of the system. In the present paper, we analyze fusion data for 64 different heavy-ion systems without using any adjustable parameter. The analyses are performed in the context of the ZPM model and using the São Paulo (SP) potential [17-19] as the bare interaction. The SP potential has been successful in describing the elastic scattering and peripheral reaction 
channels for several systems in a wide energy region, from sub-barrier to intermediate energies [18,20-35]. Therefore, the bare interaction assumed here to analyze the fusion data is also appropriate to describe the elastic scattering process. At extreme sub-barrier energies (about $20 \mathrm{MeV}$ below the barrier), the BPM underestimates the data by about eleven orders of magnitude while the results of the ZPM model agree with the data within only two orders of magnitude for most of the systems. In this context, particular characteristics in the behavior of the data for different systems can be clearly detected. We show that the predictions can be significantly improved by using adjustable parameters, but in this case the values obtained for these parameters may become unrealistic.

\section{ZPM MODEL AND SP POTENTIAL}

We have considered the scattering of a particle by a parabolic barrier ( $r$ coordinate) whose barrier height is coupled to a harmonic oscillator ( $s$ coordinate). Before the scattering the oscillator is in its ground-state and the particle has asymptotic kinetic energy $E$. The corresponding Schrödinger equation is

$$
\begin{aligned}
{\left[H_{0}+H_{o s c}\right] \psi(r, s) } & =\left(E+E^{*} / 2\right) \psi(r, s), \\
H_{0} & =-\frac{\hbar^{2}}{2 \mu} \frac{\partial^{2}}{\partial r^{2}}+V(r, s), \\
H_{o s c} & =-E^{*}\left[\sigma^{2} \frac{\partial^{2}}{\partial s^{2}}-\frac{s^{2}}{4 \sigma^{2}}\right] .
\end{aligned}
$$

The excitation energy, $E^{*}$, and the oscillator mass, $D$, are related to the standard deviation of the vibration, $\sigma$, through $D=\hbar^{2} / 2 \sigma^{2} E^{*}$. The parabolic barrier is written as

$$
\begin{aligned}
& V(r, s)=V_{B}-F s-\frac{1}{2} \mu w^{2} r^{2}, \quad-r_{0} \leqslant r \leqslant r_{0} \\
& V(r, s)=0, \quad r<-r_{0} \text { or } r>r_{0} .
\end{aligned}
$$

with $r_{0}$ defined by $\frac{1}{2} \mu w^{2} r_{0}^{2}=V_{B}$. Thus, in the regions $r<-r_{0}$ and $r>r_{0}$ the particle is in free movement. As is evident in Eq. (4), we suppose that the barrier height is coupled to the harmonic oscillator degree of freedom and the barrier curvature, $\hbar w$, is not affected by the couplings. We consider only a linear coupling which is related to the term $F s$ in Eq. (4).

The wave functions of the oscillator are

$$
\phi_{n}(s)=\frac{1}{\left(2 \pi \sigma^{2}\right)^{1 / 4}} e^{-s^{2} / 4 \sigma^{2}} \frac{H_{n}(s / \sigma \sqrt{2})}{\sqrt{n ! 2^{n}}},
$$

where $H_{n}$ are the Hermite polynomials. By expanding the wave function, $\psi(r, s)=\sum \psi_{n}(r) \phi_{n}(s)$, one obtains the coupled equations that, in the region $-r_{0} \leqslant r \leqslant r_{0}$, are written as

$$
\begin{gathered}
-\frac{\hbar^{2}}{2 \mu} \frac{d^{2} \psi_{n}(r)}{d r^{2}}+\left[V_{B}-\frac{1}{2} \mu w^{2} r^{2}\right] \psi_{n}(r) \\
+\sum_{m \neq n} V_{n m} \psi_{m}(r)=\left[E-n E^{*}\right] \psi_{n}(r),
\end{gathered}
$$

where

$$
V_{n m}=-F \sigma\left[\sqrt{n} \delta_{n, m+1}+\sqrt{m} \delta_{n, m-1}\right] .
$$

The CC equations should be solved by considering the asymptotic wave functions:

$$
\begin{aligned}
\psi_{n}\left(r \geqslant r_{0}\right) & =\delta_{n, 0} e^{-i k_{0} r}+b_{n} e^{i k_{n} r}, \\
\psi_{n}\left(r \leqslant-r_{0}\right) & =a_{n} e^{-i k_{n} r},
\end{aligned}
$$

and the total transmission coefficient is given by

$$
T=\sum_{n} T_{n}=\sum_{n} \frac{k_{n}}{k_{0}}\left|a_{n}\right|^{2} .
$$

Within an approximation for the asymptotic wave function, the total transmission coefficient can be obtained without solving the CC equations by [14]

$$
T=\sum_{n} W_{n} T_{n},
$$

where the weighting factors are

$$
W_{n}=\frac{1}{n !}\left(\frac{F \sigma}{E^{*}}\right)^{2 n} e^{-\left(F \sigma / E^{*}\right)^{2}},
$$

and the partial transmission coefficients can be obtained through the Hill-Wheeler expression [36]

$$
T_{n}=\frac{1}{1+\exp \left[2 \pi\left(V_{B}-E+\lambda_{n}\right) / \hbar w\right]},
$$

considering the corresponding eigenvalues

$$
\lambda_{n}=n E^{*}-(F \sigma)^{2} / E^{*} .
$$

The effect of the couplings is to replace the barrier height $V_{B}$ by a set of barriers $V_{B}+\lambda_{n}$, and the total transmission is given by a weighted average of the transmission for each effective barrier. In Ref. [14], the special case of only two channel problem was also addressed. In this case, one obtains the corresponding eigenvalues and weighting factors:

$$
\begin{aligned}
\lambda_{ \pm} & =\frac{1}{2}\left[E^{*} \pm \sqrt{\left(E^{*}\right)^{2}+4(F \sigma)^{2}}\right] \\
W_{ \pm} & =\frac{2(F \sigma)^{2}}{4(F \sigma)^{2}+\left(E^{*}\right)^{2} \pm E^{*} \sqrt{\left(E^{*}\right)^{2}+4(F \sigma)^{2}}} .
\end{aligned}
$$

These results can easily be generalized for a set of different vibrational degrees of freedom coupled to the parabolic barrier in a completely independent fashion. For instance, in the present paper we are going to consider the case of four degrees of freedom, where the total transmission coefficient can be obtained from

$$
\begin{aligned}
T & =\sum_{n 1} \sum_{n 2} \sum_{n 3} \sum_{n 4} W_{n 1} W_{n 2} W_{n 3} W_{n 4} T_{n}, \\
T_{n} & =\frac{1}{1+\exp \left[2 \pi \frac{\left(V_{B}-E+\lambda_{n 1}+\lambda_{n 2}+\lambda_{n 3}+\lambda_{n 4}\right)}{\hbar w}\right]} .
\end{aligned}
$$

We describe now the bare interaction, SP potential, assumed in our calculations. The SP potential has been successful in describing the elastic scattering and peripheral reaction 
channels for several heavy-ion systems in a very wide energy region [18,20-35]. In the present work, we use the SP interaction generalized for deformed nuclei [37,38]. Within this model, the nuclear interaction is connected with the folding potential through [19]:

$$
V_{N}(R, E)=V_{F}(R) e^{-4 \mathrm{v}^{2} / c^{2}},
$$

where $c$ is the speed of light, $\mathrm{v}$ is the local relative velocity between the two nuclei,

$$
\mathrm{v}^{2}(R, E)=\frac{2}{\mu}\left[E-V_{C}(R)-V_{N}(R, E)\right],
$$

and $V_{C}$ is the Coulomb potential. The folding potential depends on the matter densities of the nuclei involved in the collision:

$$
\begin{aligned}
V_{F}(R)= & \int \rho_{1}\left(\vec{r}_{1}\right) \rho_{2}\left(\vec{r}_{2}\right) \\
& \times V_{0} \delta\left(\vec{R}-\vec{r}_{1}+\vec{r}_{2}\right) d \vec{r}_{1} d \overrightarrow{r_{2}},
\end{aligned}
$$

with $V_{0}=-456 \mathrm{MeV} \mathrm{fm}^{3}$. The use of the matter densities and delta function in Eq. (22) corresponds to the zero-range approach for the folding potential, which is equivalent to the more usual procedure of using an effective nucleon-nucleon interaction with the nucleon densities of the nuclei (instead of the matter densities). In order to illustrate this point, which is extensively discussed in Refs. [19,37], in Fig. 1 we show the undeformed SP potential calculated through the zero (dashed lines) and finite (solid lines) range approaches for four systems at energies $E=V_{B 0}$. The difference between solid and dashed lines is almost indistinguishable in the figure indicating that the two approaches are in fact equivalent. However, due to the six-dimensional integral, the finite-range approach takes much more computational time in the numerical calculation of deformed potentials than the zero-range one [37]. Thus, in the present work we have assumed the zero-range in our theoretical calculations of fusion cross sections.

Hereafter, we assume a two-parameter Fermi $(2 \mathrm{pF})$ distribution to describe the deformed density of a given nucleus $i$

$$
\begin{aligned}
\rho_{i}(\vec{r}) & =\frac{\rho_{0 i}}{1+\exp \left(\frac{r-R_{i}}{a}\right)}, \\
R_{i} & =R_{0 i}\left[1+\sum_{\lambda} \beta_{\lambda i} Y_{\lambda 0}\left(\Theta_{\lambda i}\right)\right],
\end{aligned}
$$

where $\Theta_{\lambda i}$ is the angle between $\vec{r}$ and the symmetry axis of the $\lambda$ deformation. We limit the present study to $\lambda=2$ and 3 .

In an earlier paper [19], we presented an extensive systematics for the densities of heavy nuclei. We found that the radii of the matter $\left(R_{0 M}\right)$ and charge $\left(R_{0 C}\right)$ densities can be well represented by

$$
\begin{aligned}
R_{0 M} & =1.31 A^{1 / 3}-0.84 \mathrm{fm}, \\
R_{0 C} & =1.76 Z^{1 / 3}-0.96 \mathrm{fm} .
\end{aligned}
$$

The charge and matter distributions present average diffuseness values of $a=0.53 \mathrm{fm}$ and $a=0.56 \mathrm{fm}$, respectively. The quadrupole and octupole deformation parameters can be obtained based on the corresponding experimental results for the transition probabilities:

$$
B(E \lambda)=\left(\frac{3 Z e \beta_{\lambda} R_{0 C}^{\lambda}}{4 \pi}\right)^{2} .
$$

The frequencies of the $\lambda=2$ and 3 modes are connected with the excitation energies of the corresponding $2_{1}^{+}$and $3_{1}^{-}$ states. In the context of the systematics for the densities, the SP potential has no adjustable parameter. Therefore, the results of the present work for theoretical fusion cross sections represent predictions rather than data fits. This is an important feature of our model.

In the context of the BPM, the effective potential is a sum of the Coulomb, nuclear and centrifugal parts:

$$
V_{\mathrm{eff}}(R)=V_{C}(R)+V_{N}(R)+\frac{\ell(\ell+1) \hbar^{2}}{2 \mu R^{2}}
$$

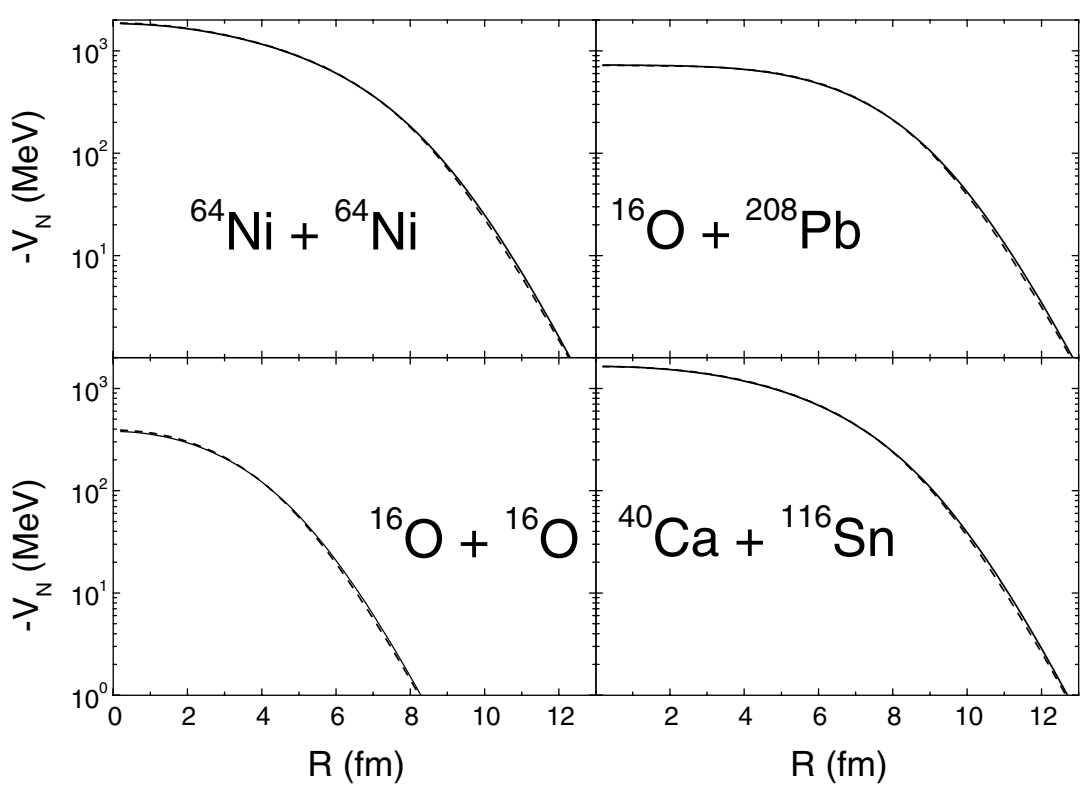

044606-3
FIG. 1. The São Paulo potential in the zero (dashed lines) and finite (solid lines) range approaches for four systems indicated in the figure. 
The BPM cross section is associated with the transmitted flux through the expression

$$
\sigma_{\mathrm{BPM}}(E)=\frac{\pi}{k^{2}} \sum(2 \ell+1) T_{\ell} .
$$

In our calculations, the sum in Eq. (29) is performed up to a maximum partial wave $\ell_{\max }$, which is the greatest $\ell$ value that results a pocket (and a barrier) in the corresponding effective potential. For $\ell$-waves with effective barrier heights $\left(V_{B \ell}\right)$ below the center of mass energy, we have approximated the effective potential by a parabola with curvature:

$$
\hbar \omega_{\ell}=\left|\frac{\hbar^{2}}{\mu} \frac{d^{2} V_{\mathrm{eff}}}{d R^{2}}\right|_{R_{B \ell}}^{1 / 2},
$$

where $R_{B \ell}$ is the barrier radius. On the other hand, for $\ell$-waves with $V_{B \ell} \gg E$, the approximation of the effective potential by a parabolic barrier is not appropriate due to the tail of the Coulomb potential, and the calculation of transmission coefficients through the Hill-Wheeler expression using the curvature provided by Eq. (30) is not accurate anymore. Thus, in this regime, we define $\hbar w$ by another form. For $\ell$-waves with $V_{B \ell}>E$ we have adopted the more appropriated WKB method:

$$
\begin{aligned}
T_{\ell} & =\left[1+\exp \left(S_{\ell}\right)\right]^{-1}, \\
S_{\ell} & =\int_{R_{1}}^{R_{2}} \sqrt{\frac{8 \mu}{\hbar^{2}}\left[V_{\mathrm{eff}}(R)-E\right]} d R,
\end{aligned}
$$

where $R_{1}$ and $R_{2}$ are the classical turning points. In this case, we obtain the barrier curvature that, within the context of the Hill-Wheeler expression, would provide the same value for the transmission coefficient as obtained with the WKB calculations for the undeformed effective potential:

$$
\hbar w_{\ell}=\frac{2 \pi\left(V_{B \ell}-E\right)}{S_{\ell}} .
$$

Then, we use this value in Eq. (19) which contains the effect of the couplings. With this method, we assume that even for $E \ll$ $V_{B \ell}$ the effective potential can be described by a parabolic barrier.

Figure 2 shows a schematic representation of the collision of two deformed nuclei, where $\theta$ would represent the direction of the symmetry axis of the nucleus and $\vec{R}$ connects the center of mass of both nuclei. Due to the short range of the nuclear interaction, the barrier height depends practically only on the

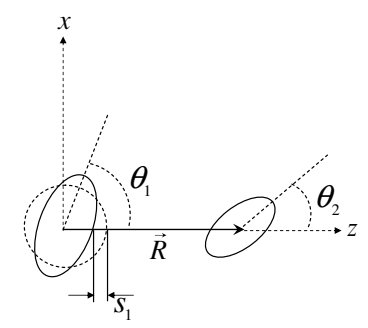

FIG. 2. Schematic representation of the collision between two deformed nuclei. The $s$ coordinate represents the variation of the nuclear radius relative to the spherical shape in the direction of $\vec{R}$. coordinate $s$ represented in this same figure. Different sets of $\theta$ and $\beta$ values that result in the same $s$ value also provide very similar $V_{B \ell}$ values. Thus, we have obtained coupling amplitudes by considering the derivative of the barrier height relative to the $s$ coordinate:

$$
F_{\lambda i}(\ell)=-\frac{\partial V_{B \ell}}{\partial s_{\lambda i}},
$$

where $i$ could represent nucleus 1 or 2 . Within the vibrational model, the standard deviation of the $s$ coordinate is connected with the $\beta$ value through

$$
\sigma_{\lambda i}=\frac{\beta_{\lambda i} R_{0 i}}{\sqrt{4 \pi}} .
$$

In this context, the collision of two deformed nuclei involves four vibrational coordinates $\left(s_{\lambda i}\right)$ corresponding to the quadrupole and octupole modes of both nuclei. As a further approximation, we have assumed that the $F_{\lambda i}$ are independent parameters and the partial derivative involved in Eq. (34) has been calculated with respect to a spherical shape.

The Coulomb and nuclear deformation parameters may not have the same value, because they are related to different (charge and matter) distributions. However, to avoid the use of adjustable parameters, we have assumed the same value for both deformations. This procedure is in fact quite reasonable because the nuclear and Coulomb deformations are expected to have similar values. The $\beta_{2}$ and $\beta_{3}$ values were obtained from Eq. (27), through transition amplitudes from the systematics for even-even nuclei presented in Refs. [39,40].

In the context presented here, the ZPM model describes, in an approximate form, the couplings of excited states related to complete bands of quadrupole and octupole modes of vibration, corresponding to a large number of couplings to inelastic states. However, no couplings to transfer channels are included in our calculations. Some of the nuclei involved in this work could be better represented by the rotational model rather than the vibrational one. On the other hand, the standard deviation $\sigma_{\lambda}$ of rotational distributions is related to the corresponding $\beta_{\lambda}$ value through the same expression that is valid for vibrational distributions, Eq. (35). In fact, within the rotational model one obtains

$$
\begin{aligned}
\sigma_{\lambda}^{2} & =\int_{0}^{\pi}\left(R_{i}-R_{0 i}\right)^{2} f(\theta) d \theta \\
& =\int_{0}^{\pi}\left[\beta_{\lambda} R_{0 i} Y_{\lambda 0}(\theta)\right]^{2} f(\theta) d \theta,
\end{aligned}
$$

where $f(\theta)=1 / 2 \sin (\theta)$ is the distribution probability of $\theta$, which inserted in Eq. (36) results in Eq. (35). Nevertheless, the shapes of the distribution for the $s$ coordinate in the rotational and vibrational modes are different. Even so, we have assumed the ZPM model for all systems with the aim of investigating the limits of this model in accounting for fusion data.

Within the ZPM model, we neglect the fact that the excitation of each surface mode corresponds to a finite transfer of angular momentum. The calculations would become much more complicated if such effects were included. This procedure has already been adopted in other works (see, e.g., [41]) because angular momentum transfer effects have little 
influence on total cross sections for heavy colliding systems, as discussed in Ref. [42].

\section{COMPARISON BETWEEN THEORETICAL AND EXPERIMENTAL FUSION CROSS SECTIONS}

In Table I, we present the nuclei involved in the systems studied in the present work, with the corresponding $\beta$ and $E^{*}$ values for the quadrupole and octupole modes. Figures 3-21 and 24 present the fusion data (from references provided in the

TABLE I. The table presents the values of the quadrupole $\left(\beta_{2}\right)$ and octupole $\left(\beta_{3}\right)$ deformation parameters, and also the corresponding excitation energies for the nuclei studied in this work.

\begin{tabular}{|c|c|c|c|c|}
\hline nucleus & $\beta_{2}$ & $E_{2}^{*}(\mathrm{MeV})$ & $\beta_{3}$ & $E_{3}^{*}(\mathrm{MeV})$ \\
\hline${ }^{12} \mathrm{C}$ & 0.89 & 4.44 & 1.55 & 9.64 \\
\hline${ }^{16} \mathrm{O}$ & 0.50 & 6.92 & 1.20 & 6.13 \\
\hline${ }^{18} \mathrm{O}$ & 0.54 & 1.98 & 1.10 & 5.10 \\
\hline${ }^{28} \mathrm{Si}$ & 0.50 & 1.78 & 0.55 & 6.88 \\
\hline${ }^{30} \mathrm{Si}$ & 0.41 & 2.24 & 0.41 & 5.49 \\
\hline${ }^{32} \mathrm{~S}$ & 0.38 & 2.23 & 0.70 & 5.01 \\
\hline${ }^{36} \mathrm{~S}$ & 0.32 & 3.29 & 0.56 & 4.19 \\
\hline${ }^{40} \mathrm{Ar}$ & 0.32 & 1.46 & 0.58 & 3.68 \\
\hline${ }^{40} \mathrm{Ca}$ & 0.14 & 3.90 & 0.51 & 3.74 \\
\hline${ }^{48} \mathrm{Ca}$ & 0.13 & 3.83 & 0.34 & 4.51 \\
\hline${ }^{48} \mathrm{Ti}$ & 0.32 & 0.98 & 0.26 & 3.36 \\
\hline${ }^{58} \mathrm{Ni}$ & 0.21 & 1.45 & 0.24 & 4.48 \\
\hline${ }^{64} \mathrm{Ni}$ & 0.22 & 1.35 & 0.26 & 3.56 \\
\hline${ }^{68} \mathrm{Zn}$ & 0.24 & 1.08 & 0.30 & 2.75 \\
\hline${ }^{70} \mathrm{Ge}$ & 0.26 & 1.04 & 0.33 & 2.56 \\
\hline${ }^{76} \mathrm{Ge}$ & 0.32 & 0.56 & 0.19 & 2.69 \\
\hline${ }^{86} \mathrm{Kr}$ & 0.17 & 1.56 & 0.19 & 3.10 \\
\hline${ }^{90} \mathrm{Zr}$ & 0.10 & 2.19 & 0.25 & 2.75 \\
\hline${ }^{92} \mathrm{Zr}$ & 0.12 & 0.93 & 0.21 & 2.34 \\
\hline${ }^{96} \mathrm{Zr}$ & 0.10 & 1.75 & 0.36 & 1.90 \\
\hline${ }^{92} \mathrm{Mo}$ & 0.12 & 1.51 & 0.19 & 2.85 \\
\hline${ }^{94} \mathrm{Mo}$ & 0.17 & 0.87 & 0.18 & 2.53 \\
\hline${ }^{98} \mathrm{Mo}$ & 0.19 & 0.79 & 0.27 & 2.02 \\
\hline${ }^{100} \mathrm{Mo}$ & 0.27 & 0.54 & 0.27 & 1.91 \\
\hline${ }^{102} \mathrm{Ru}$ & 0.28 & 0.48 & 0.17 & 2.04 \\
\hline${ }^{104} \mathrm{Ru}$ & 0.32 & 0.36 & 0.16 & 1.97 \\
\hline${ }^{108} \mathrm{Pd}$ & 0.28 & 0.43 & 0.20 & 2.05 \\
\hline${ }^{110} \mathrm{Pd}$ & 0.30 & 0.37 & 0.19 & 2.04 \\
\hline${ }^{112} \mathrm{Sn}$ & 0.14 & 1.26 & 0.15 & 2.36 \\
\hline${ }^{116} \mathrm{Sn}$ & 0.13 & 1.29 & 0.18 & 2.27 \\
\hline${ }^{122} \mathrm{Sn}$ & 0.12 & 1.14 & 0.15 & 2.49 \\
\hline${ }^{124} \mathrm{Sn}$ & 0.11 & 1.13 & 0.13 & 2.60 \\
\hline${ }^{144} \mathrm{Sm}$ & 0.10 & 1.66 & 0.17 & 1.81 \\
\hline${ }^{148} \mathrm{Sm}$ & 0.16 & 0.55 & 0.19 & 1.16 \\
\hline${ }^{152} \mathrm{Sm}$ & 0.35 & 0.12 & 0.12 & 1.04 \\
\hline${ }^{154} \mathrm{Sm}$ & 0.39 & 0.08 & 0.10 & 1.01 \\
\hline${ }^{182} \mathrm{~W}$ & 0.28 & 0.10 & 0.06 & 1.37 \\
\hline${ }^{186} \mathrm{~W}$ & 0.25 & 0.12 & 0.07 & 1.04 \\
\hline${ }^{192} \mathrm{Os}$ & 0.19 & 0.21 & 0.07 & 1.34 \\
\hline${ }^{194} \mathrm{Pt}$ & 0.16 & 0.33 & 0.07 & 1.43 \\
\hline${ }^{208} \mathrm{~Pb}$ & 0.06 & 4.08 & 0.13 & 2.61 \\
\hline${ }^{238} \mathrm{U}$ & 0.32 & 0.05 & 0.10 & 0.73 \\
\hline
\end{tabular}

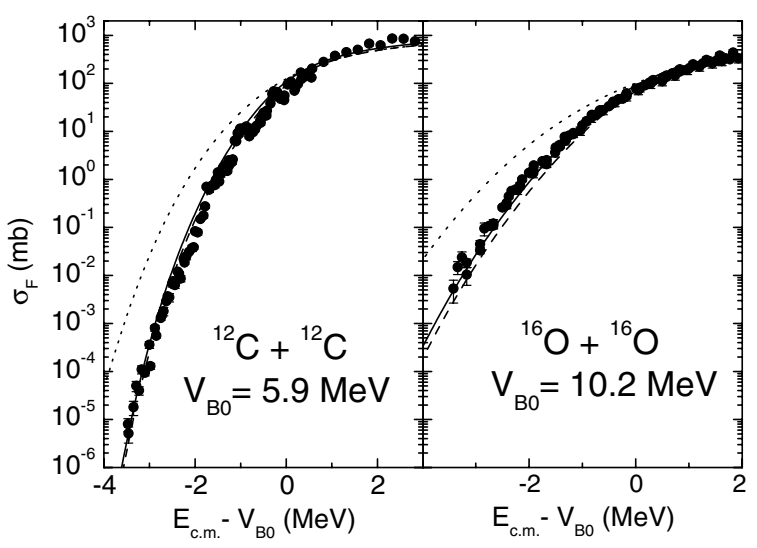

FIG. 3. Fusion data (from Refs. [44-52]) and corresponding undeformed BPM (dashed lines) and ZPM (solid lines) theoretical cross sections for the ${ }^{12} \mathrm{C}+{ }^{12} \mathrm{C}$ and ${ }^{16} \mathrm{O}+{ }^{16} \mathrm{O}$ systems. The dotted lines represent the results of the FA, i.e. those obtained considering vanishing excitation energies. The s-wave barrier heights of the undeformed potential are presented in the figure.

captions) and corresponding results of the undeformed BPM (dashed lines) and the ZPM (solid lines) calculations. The energy scale is represented relative to the undeformed s-wave barrier height $\left(V_{B 0}\right)$. With exception of Figs. 3, 14 and 21, the figures have the same scale of energy and cross section. This procedure makes simple the comparison among results of different systems.

In Fig. 3 we present the lightest systems studied in the present work. In the case of ${ }^{12} \mathrm{C}+{ }^{12} \mathrm{C}$, the BPM and ZPM results are almost indistinguishable and agree quite well with the data in the entire energy region corresponding to about nine orders of magnitude in cross sections. For ${ }^{16} \mathrm{O}+{ }^{16} \mathrm{O}$, the ZPM cross sections are slightly larger than the BPM predictions and appropriately approach the data at sub-barrier energies. Despite the large $\beta$ values of the ${ }^{12} \mathrm{C}$ and ${ }^{16} \mathrm{O}$ nuclei (see Table I), the ZPM and BPM predictions are very similar and almost no effect of the couplings is observed. This is because the coupling strength $F$ for light systems is small and the excitation energies for these nuclei are large. To illustrate this point, the dotted lines in Fig. 3 represent the results of the FA, i.e., those obtained considering $E^{*}=0$. By comparing the solid and dotted lines, one can notice the significant effect of the excitation energy.

Figure 4 presents fusion data for the ${ }^{12} \mathrm{C}+{ }^{92} \mathrm{Zr}$ and ${ }^{12} \mathrm{C}$, ${ }^{18} \mathrm{O},{ }^{32} \mathrm{~S}+{ }^{208} \mathrm{~Pb}$ systems. Large enhancements of the data relative to the BPM cross sections can be observed, and the ZPM results are in much better agreement with the data. The heavier systems present larger enhancements than the lighter ones, as was already reported many years ago [2]. This behavior is due to the increasing values of couplings strengths $(F)$ for heavier systems. However, this in fact is not a general behavior of the heavy-ion fusion, and a few exceptions can be found. For instance, in Fig. 5 the enhancement for ${ }^{16} \mathrm{O}+{ }^{208} \mathrm{~Pb}$ is in fact slightly smaller than those for the lighter ${ }^{16} \mathrm{O}+{ }^{182,186} \mathrm{~W}$ systems. On the other hand, the ZPM cross sections are in good agreement with the data (see Fig. 5). This behavior is related to the larger $\beta_{2}$ (and smaller $E_{2}^{*}$ ) 


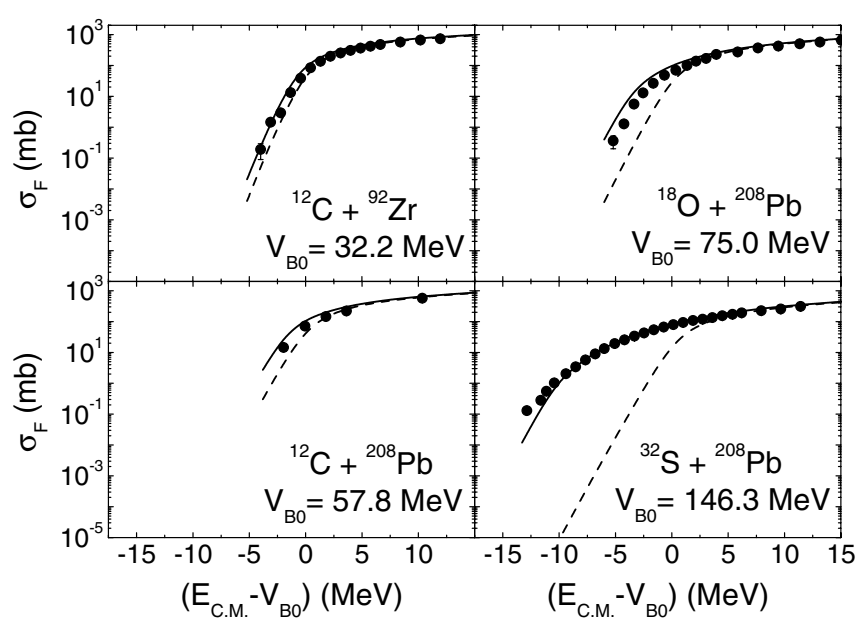

FIG. 4. The same as Fig. 3, for the systems indicated in the figure. The data are from Refs. [53-56].

values of the ${ }^{182,186} \mathrm{~W}$ in comparison with the ${ }^{208} \mathrm{~Pb}$ (see Table I).

Figure 6 shows systems with the ${ }^{16} \mathrm{O}$ projectile on targets that correspond to different samarium isotopes. Despite the similar region of mass, significant differences in the magnitude of the enhancements (relative to BPM) are observed indicating strong effects of the structure of the target nuclei. These systems were analyzed in Ref. [12], where for the first time the zero point motion of the nuclear surface was applied in the study of heavy-ion fusion. In that work, the effect of the excitation energy was not considered and an adjustable parameter related to the bare potential was used to fit the data. Clearly, the present ZPM results also account for these effects of structure due to the very different $\beta_{2}$ and $E_{2}^{*}$ values of the samarium isotopes (see Table I). The effects of shell structure observed in $\beta_{2}$ but not in $\beta_{3}$ are well understood microscopically. To lowest order, collective quadrupole transitions are constructed of a linear combination of $J=2$, positive parity particle-hole excitations. Within open shells, such excitations involve states within the same

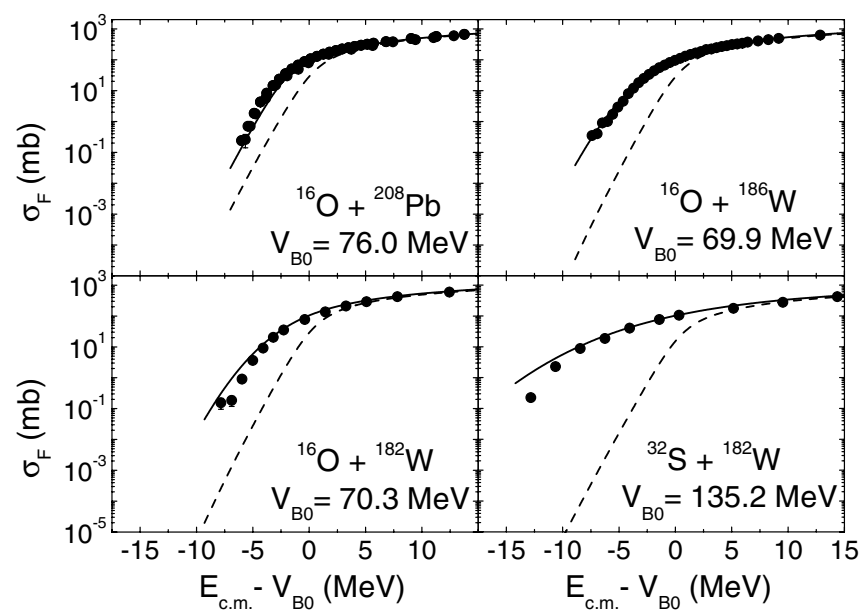

FIG. 5. The same as Fig. 3, for the systems indicated in the figure. The data are from Refs. [55,57-60].

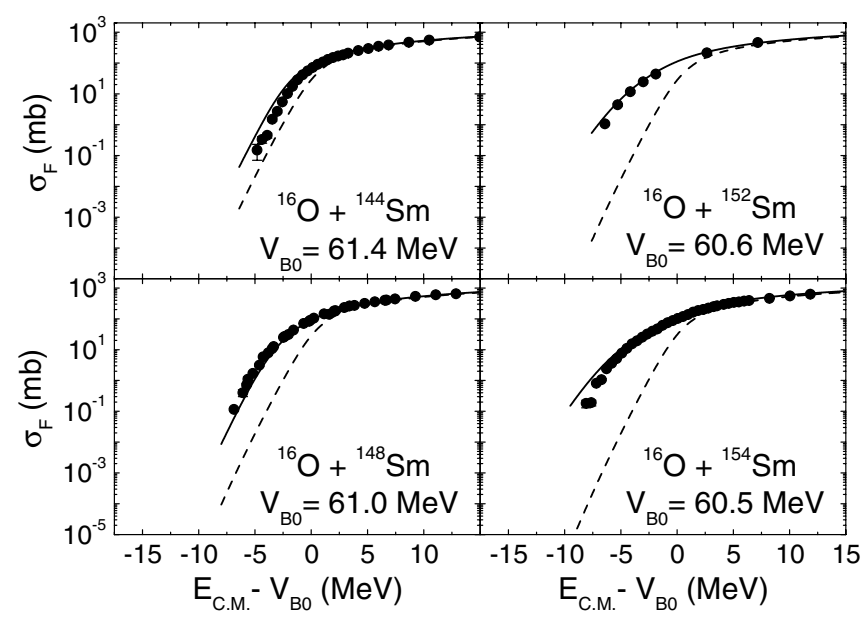

FIG. 6. The same as Fig. 3, for the systems indicated in the figure. The data are from Refs. [57,61].

major shell. At shell closures, however, the particle state must be one from two major shells above the closed shell, which inhibits the excitation due to the large energy involved. $\beta_{2}$ is thus large where a shell is filling and tend to zero at its closure. Collective octupole transitions, on the other hand, are a linear combination of $J=3$, negative parity particle-hole excitations, which involve a hole state from one shell and a particle state from the adjacent shell. Since such combinations always exist, independently of shell closures, little structure is observed in $\beta_{3}$.

Figure 7 presents systems that involve ${ }^{28} \mathrm{Si}$ as projectile. Figures $8-10$ show systems with ${ }^{32,36} \mathrm{~S}$. Figures 11,12 , and 13 correspond to systems involving ${ }^{40,48} \mathrm{Ca}$ and ${ }^{40} \mathrm{Ar}$. The overall agreement between data and ZPM results is good and comparable with the results presented in the earlier Figs. 3-6.

In Fig. 14 we present the ${ }^{40} \mathrm{Ar}+{ }^{144} \mathrm{Sm}$. The contributions of the evaporation residues (open circles) and fusion-fission (semiclosed circles) processes to the total fusion cross sections (closed circles) are also presented. For quite heavy systems the fusion-fission process is responsible for important part of

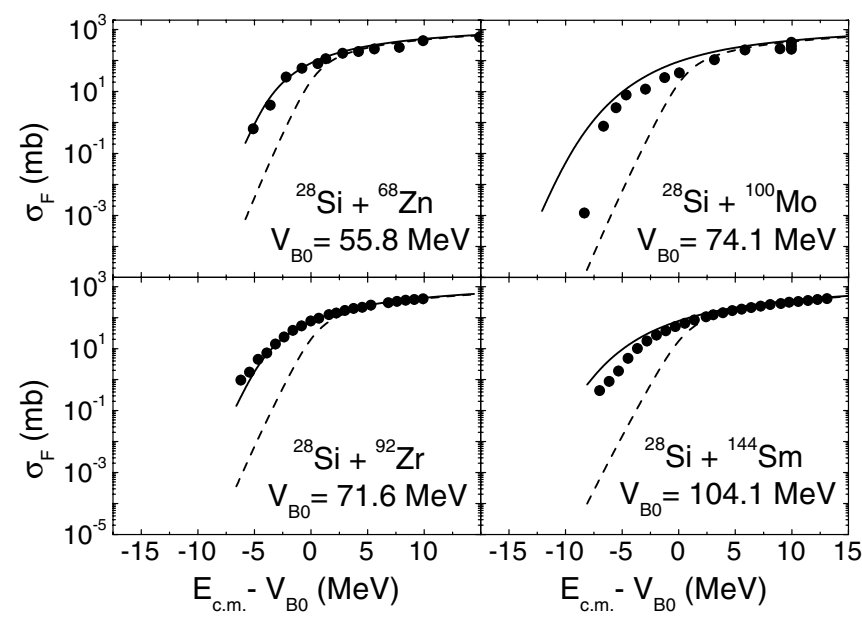

FIG. 7. The same as Fig. 3, for the systems indicated in the figure. The data are from Refs. [53,62-64]. 


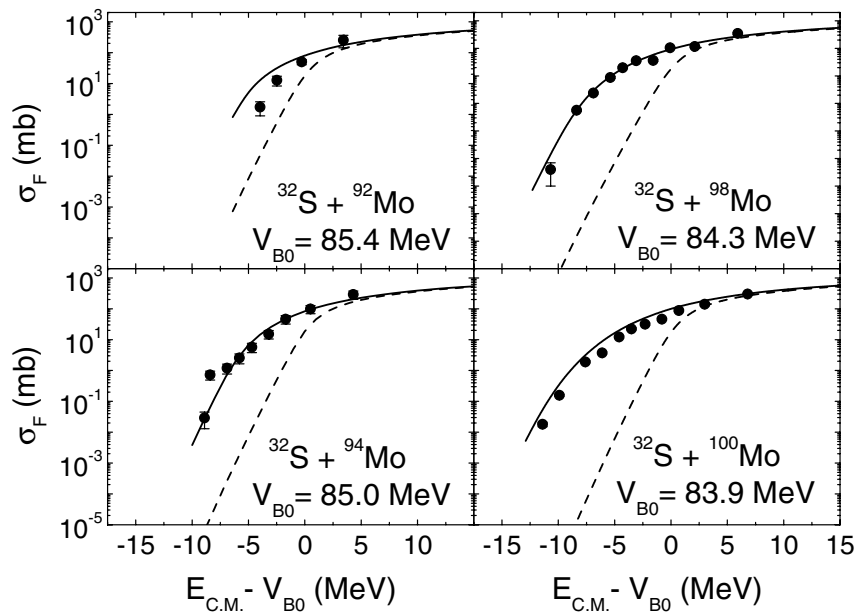

FIG. 8. The same as Fig. 3, for the systems indicated in the figure. The data are from Ref. [65].

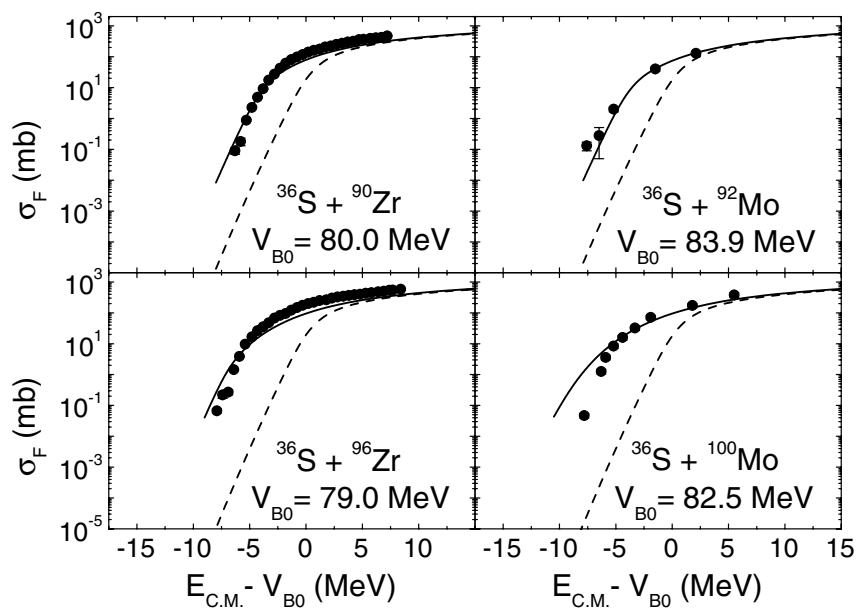

FIG. 9. The same as Fig. 3, for the systems indicated in the figure. The data are from Refs. [65,66].

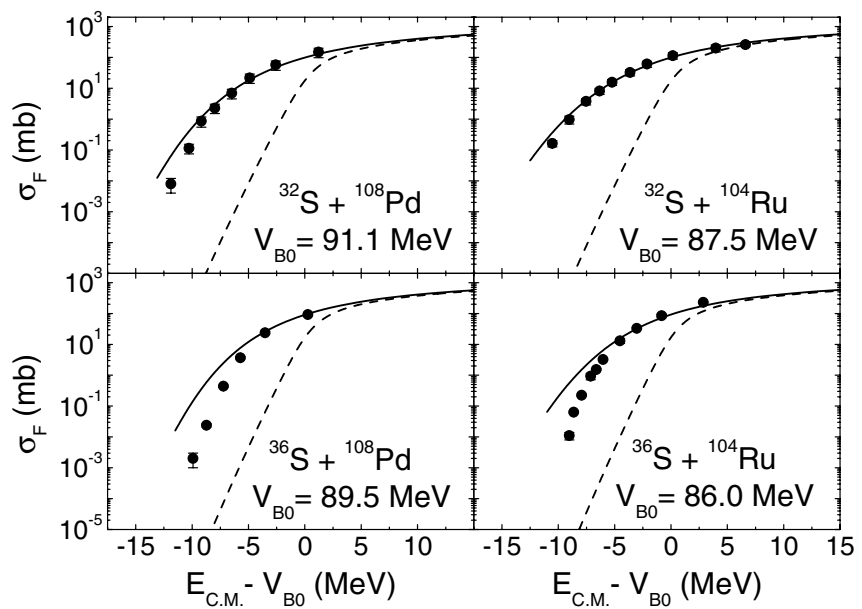

FIG. 10. The same as Fig. 3, for the systems indicated in the figure. The data are from Refs. [65].

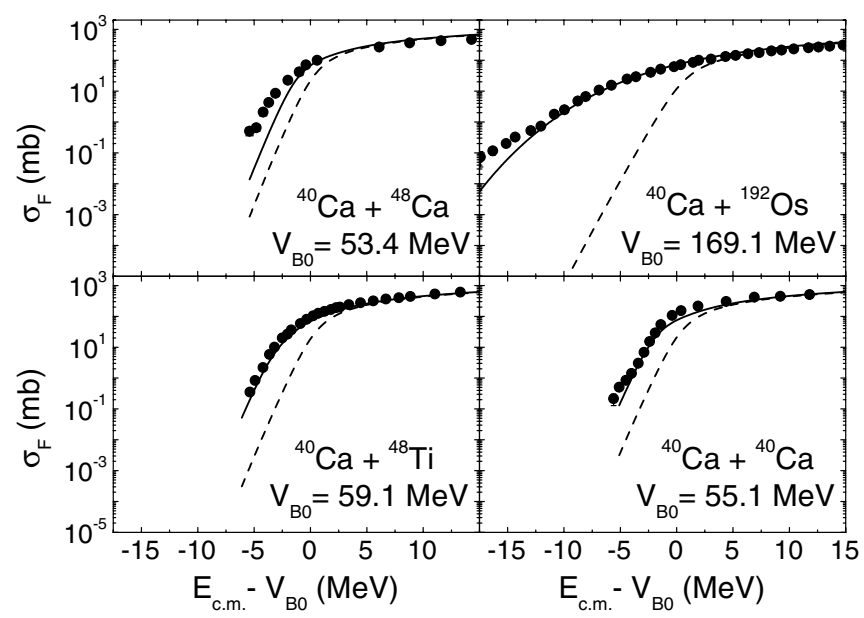

FIG. 11. The same as Fig. 3, for the systems indicated in the figure. The data are from Refs. $[67,69,70]$.

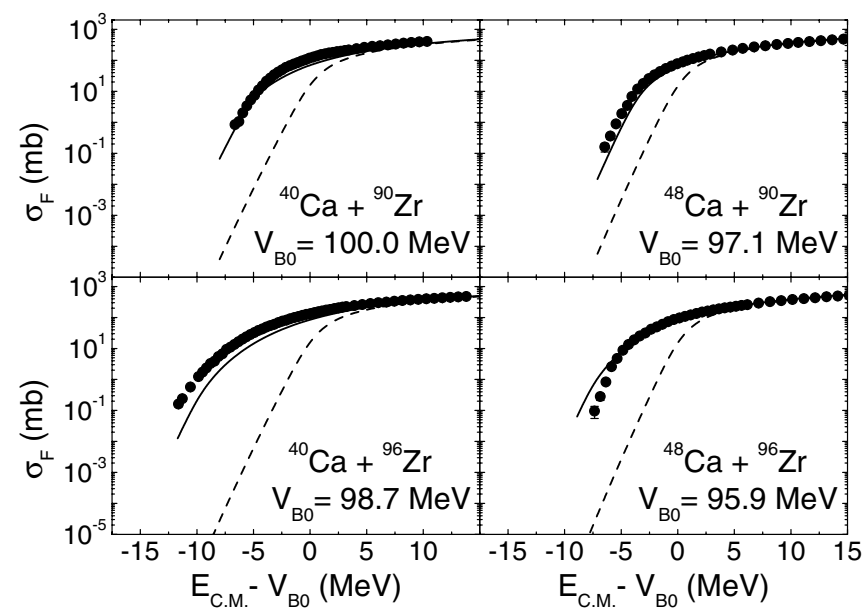

FIG. 12. The same as Fig. 3, for the systems indicated in the figure. The data are from Refs. [6,71].

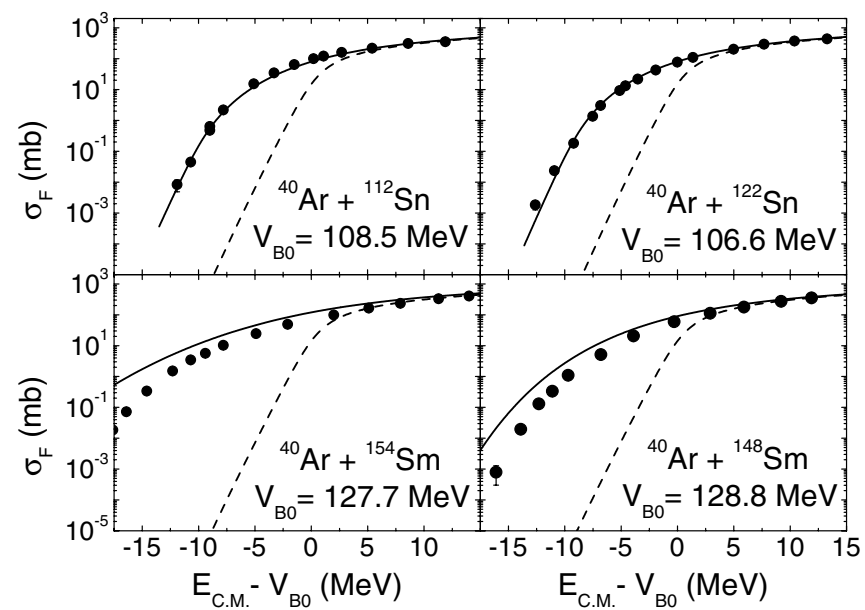

FIG. 13. The same as Fig. 3, for the systems indicated in the figure. The data are from Ref. [72]. 


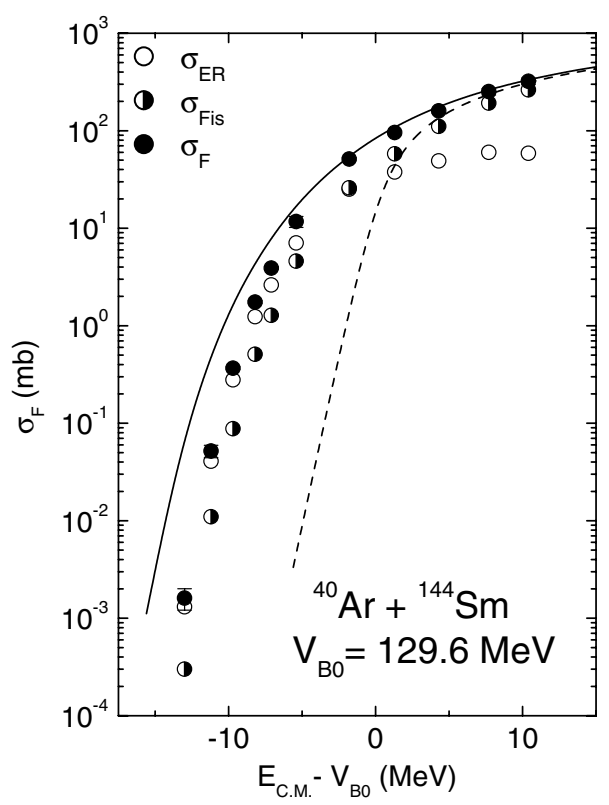

FIG. 14. Total fusion cross section data (closed circles) for the ${ }^{40} \mathrm{Ar}+{ }^{144} \mathrm{Sm}$ system (data from Ref. [72]). The contributions of evaporation residue (open circles) and fusion-fission (semi-closed circles) for the total fusion are also presented. The dashed and solid lines correspond to BPM and ZPM cross sections, respectively.

the total fusion cross section. As expected, the ZPM results represent the total fusion (see Fig. 14) since these results are associated to the complete absorption of flux arising from the barrier tunneling. As further examples, in Fig. 15 several systems involving the ${ }^{84} \mathrm{Kr}$ nucleus are presented. For these systems only the evaporation residue cross sections have been measured. The comparison between data and theoretical predictions indicates that the fusion-fission contribution become more important for heavier systems. Indeed, while for the germanium targets this contribution does not seem to be significant in the complete energy region, for ${ }^{92}$ Mo clearly

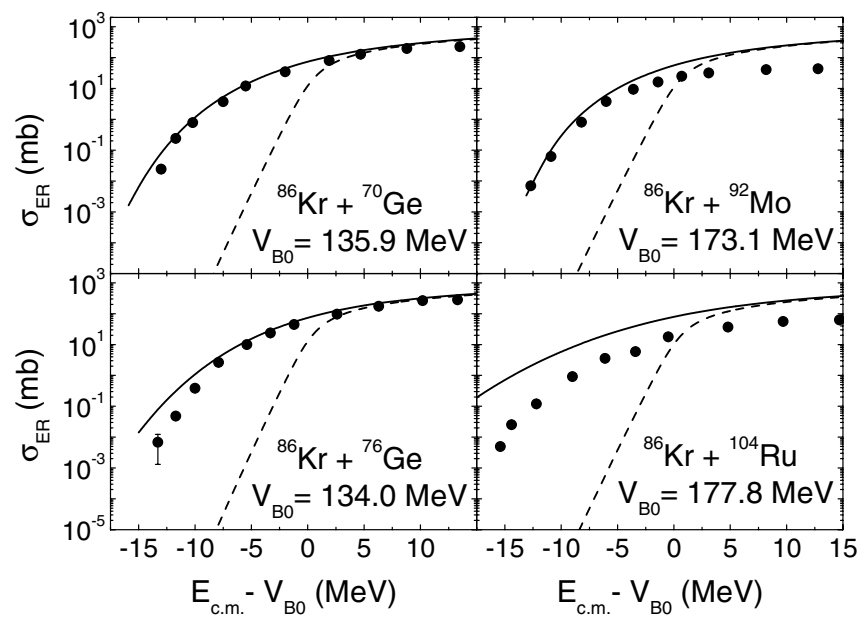

FIG. 15. Evaporation residue cross sections for several systems involving ${ }^{86} \mathrm{Kr}$ as projectile (data from Ref. [73]). The dashed and solid lines correspond to BPM and ZPM results, respectively.

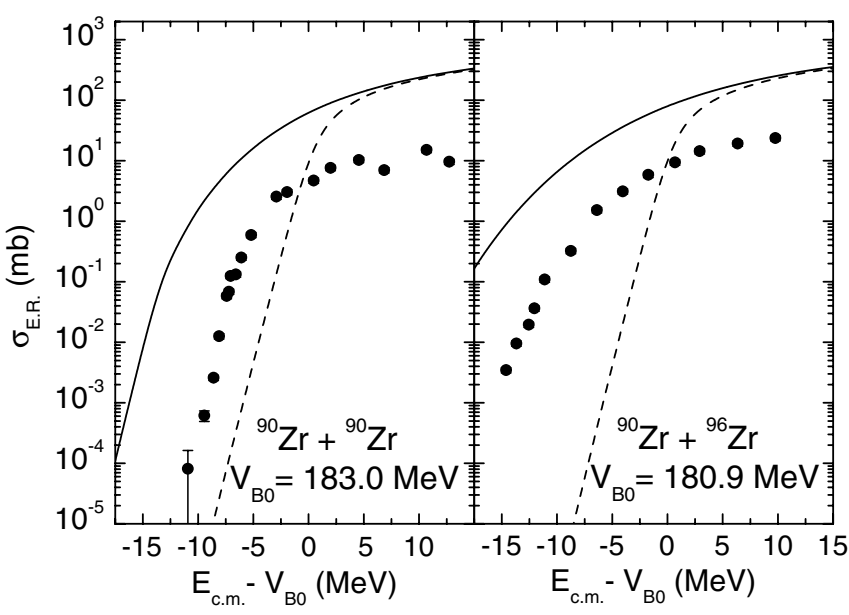

FIG. 16. The same as Fig. 15, for the systems indicated in the figure (data from Ref. [74]).

the fusion-fission should be important at the region above the barrier. In fact, for all systems presented earlier (Figs. 3-14), the ZPM and also the BPM predictions agree very well with the data for above barrier energies, but for ${ }^{84} \mathrm{Kr}+{ }^{92} \mathrm{Mo}$ this behavior is not observed. For ${ }^{84} \mathrm{Kr}+{ }^{104} \mathrm{Ru}$ the fusion-fission process could be important also at sub-barrier energies (see Fig. 15). For symmetric systems, this effect is even more emphasized, as can be observed in Fig. 16 for ${ }^{90} \mathrm{Zr}+{ }^{90,96} \mathrm{Zr}$.

In Figs. 17 and 18 other heavy systems are presented. The corresponding ZPM cross sections represent well the behavior of the data, except for ${ }^{64} \mathrm{Ni}+{ }^{100} \mathrm{Mo}$ and ${ }^{58} \mathrm{Ni}+{ }^{112} \mathrm{Sn}$ (Fig. 18). In this latter case the lowest energy datum has fusion cross section about three orders of magnitude larger than the corresponding theoretical prediction. On the other hand, for the similar ${ }^{58} \mathrm{Ni}+{ }^{124} \mathrm{Sn}$ system (Fig. 18) the ZPM cross sections are in good accordance with the data. We discuss the possible sources of these significant differences for similar systems in the next section.

Figure 19 presents fusion data and corresponding BPM and ZPM results for the ${ }^{16} \mathrm{O}+{ }^{238} \mathrm{U}$ and ${ }^{28} \mathrm{Si}+{ }^{208} \mathrm{~Pb}$ systems.

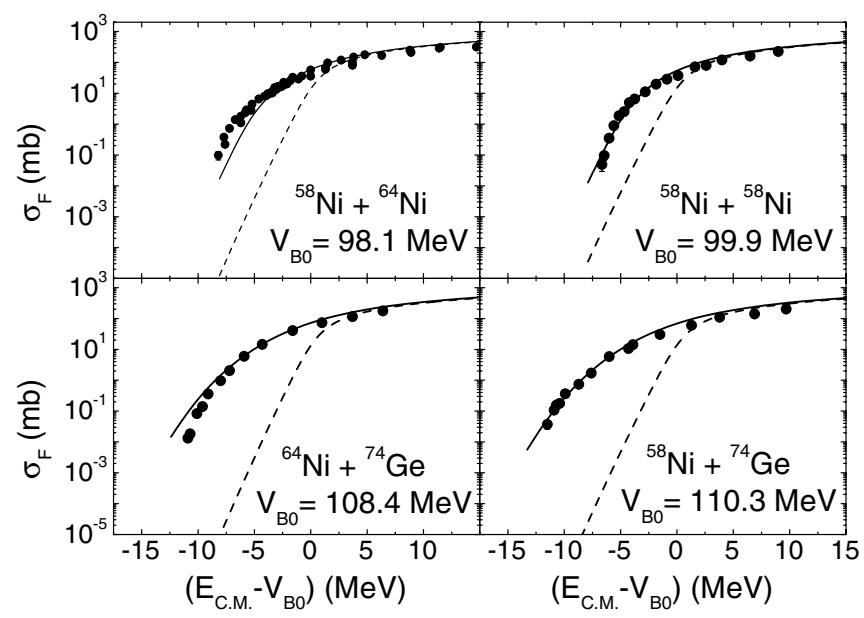

FIG. 17. The same as Fig. 3, for the systems indicated in the figure. The data are from Refs. $[63,75,76]$. 


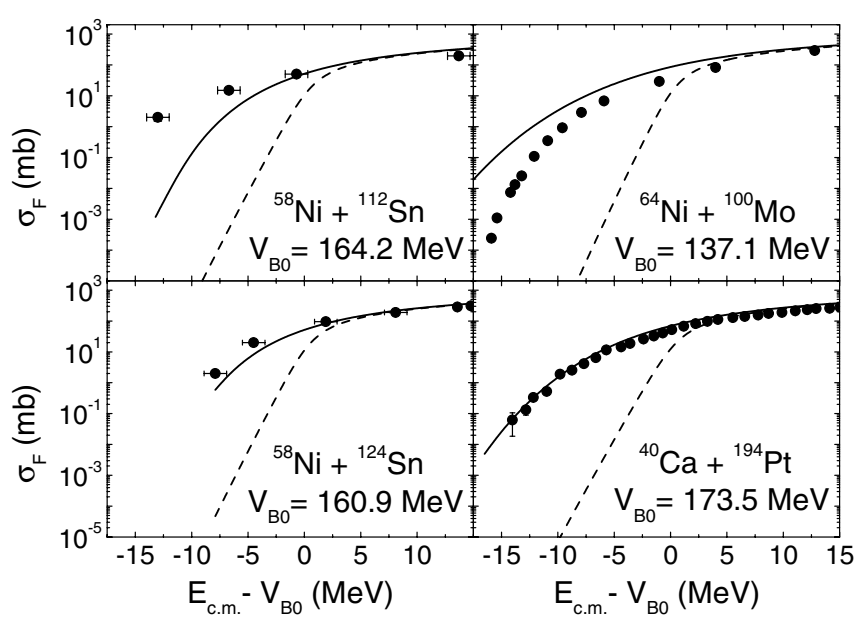

FIG. 18. The same as Fig. 3, for the systems indicated in the figure. The data are from Refs. $[11,70,77,78]$. In the case of the ${ }^{58} \mathrm{Ni}+{ }^{112,124} \mathrm{Sn}$ systems, experimental uncertainties of $1 \mathrm{MeV}$ in the corresponding energy values are indicated in the figure.

The ZPM cross sections are in good agreement with the data. The experimental and theoretical barrier distributions (BD) are also presented in the upper panel of the figure. Clearly, the effect of the couplings makes the ZPM distributions (solid lines) wider than those corresponding to the BPM (dashed lines), resulting in a quite reasonable agreement between experimental and theoretical BD. Two other examples of BD are showed in Fig. 20. In these cases, the experimental BD present complicated structures while the theoretical BD give smooth curves that correspond to the average behavior of the experimental BD.

Figure 21 presents the ${ }^{64} \mathrm{Ni}+{ }^{64} \mathrm{Ni}$ system. Besides the BPM and ZPM calculations, for this system we have also obtained theoretical cross sections through usual CC calculations performed with the FRESCO code [43]. These CC calculations included inelastic couplings only to the $2^{+}$and $3^{-}$states

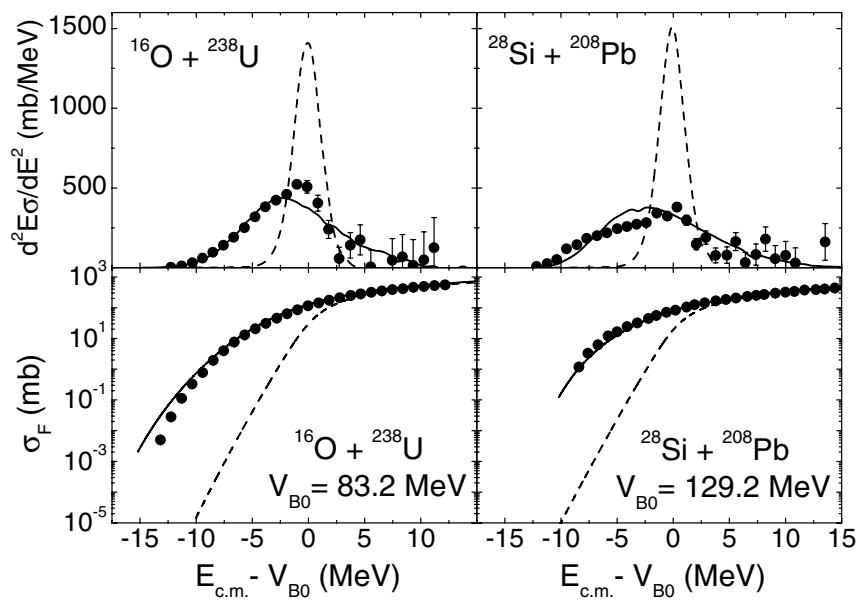

FIG. 19. Bottom: fusion cross section data (from Refs. $[79,80]$ ) for the ${ }^{16} \mathrm{O}+{ }^{238} \mathrm{U}$ and ${ }^{28} \mathrm{Si}+{ }^{208} \mathrm{~Pb}$ systems. Top: experimental barrier distributions for the same systems. The dashed and solid lines in the figure represent BPM and ZPM results, respectively.

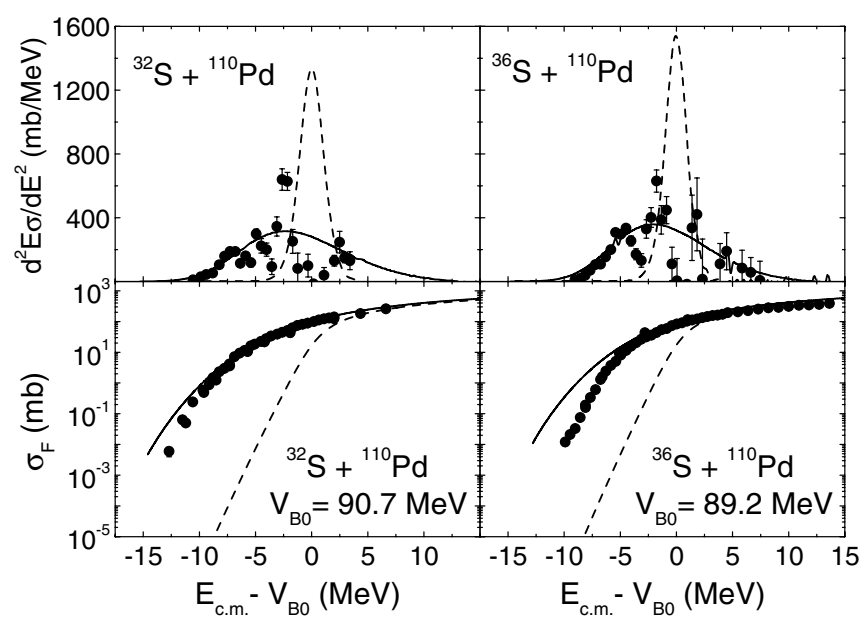

FIG. 20. The same as Fig. 19, for the systems indicated in the figure (data from Refs. [65,81]).

of the ${ }^{64} \mathrm{Ni}$. The results of these $\mathrm{CC}$ calculations were not presented for $E_{\text {c.m. }}<87 \mathrm{MeV}$ (see Fig. 21), because for very low energies the numerical calculations do not converge nor present reliable cross sections. Such numerical problems are related with the normal progressive radial method for solving the $\mathrm{CC}$ equations that fails when too many channels are locally closed, resulting in inaccurate integration through classically forbidden region $\left(E \ll V_{\text {eff }}\right)$ for such channels. In FRESCO, the fusion is calculated as the difference between the reaction cross section and the sum of the outgoing cross sections. This

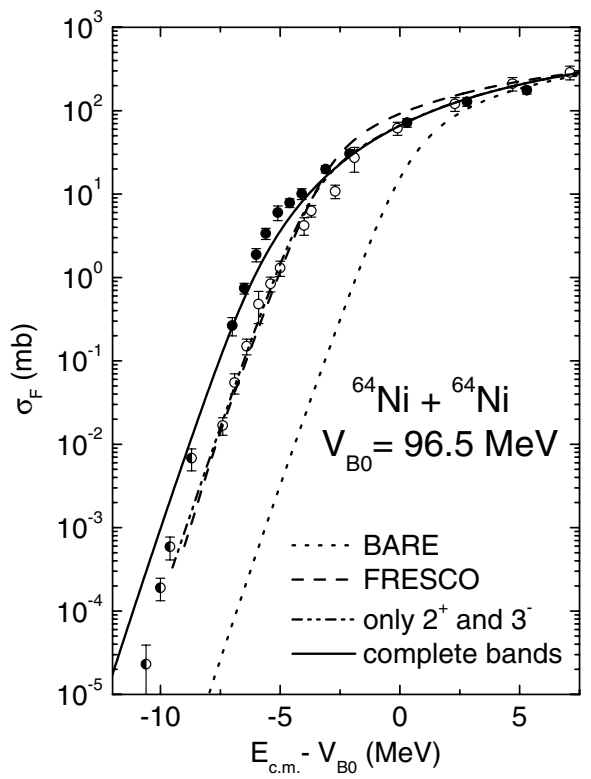

FIG. 21. Fusion data (from Refs. $[8,63,68]$ ) and corresponding undeformed BPM (dashed lines) and ZPM (solid lines) theoretical cross sections for the ${ }^{64} \mathrm{Ni}+{ }^{64} \mathrm{Ni}$ system. The other lines indicate $\mathrm{CC}$ calculations with the FRESCO code and with the model represented by equations (16) and (17), where only the couplings to the $2^{+}$and $3^{-}$states where considered. There are different data sets (represented by different symbols) obtained in different works, with cross sections that differ each other by a factor of about 3 for a few energies. 

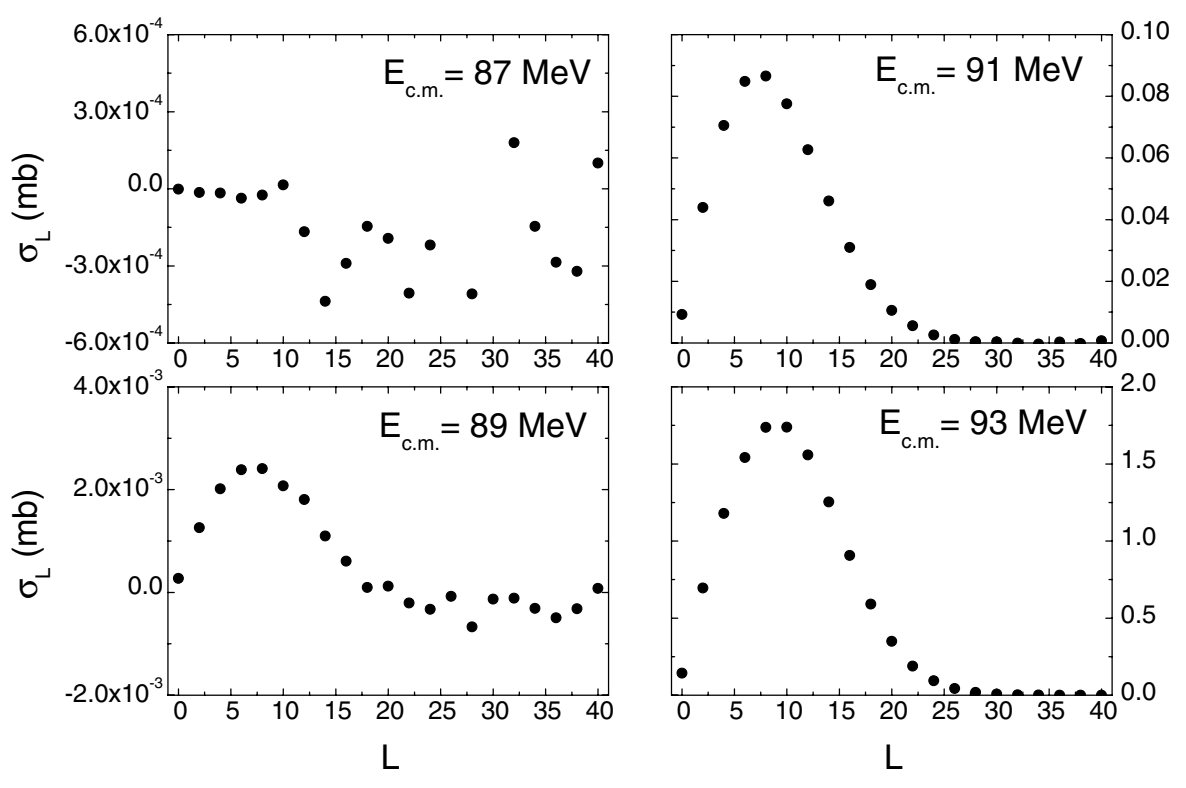

FIG. 22. Partial fusion cross sections at four energies as obtained from $\mathrm{CC}$ calculations for the ${ }^{64} \mathrm{Ni}+{ }^{64} \mathrm{Ni}$ system. means that, at low energies when the reaction and outgoing cross sections are nearly equal, the fusion is a small difference of large numbers and hence cannot be calculated accurately. To illustrate this point, in Fig. 22 we show the partial contribution of each $\ell$-wave to the total CC fusion cross section in different energies. For $E=89 \mathrm{MeV}$, the behavior of the partial fusion cross section becomes oscillatory for $\ell \geqslant 18$, including some negative values for particular $\ell$ values, which is an indication that numerical problems have occurred. Even so, for this energy a reliable value for the total fusion can be obtained if one consider the integration of partial waves only up to $\ell=18$. For the lowest energy of Fig. 22, $E=87 \mathrm{MeV}$, the oscillations are much larger, with several negative values for the partial cross section, and there is no form to determine a reliable total fusion cross section. There are other ways to solve the CC equations, such as using R-matrix expansions, and also other way to obtain fusion cross section, through the integral of the square of the multichannel wave function with the imaginary potentials (both diagonal and nondiagonal), which might avoid this problem. An advantage of the present model is the absence of numerical problems in such conditions. In fact, the ZPM calculations could be performed down to very low energies (see Fig. 21). On the other hand, the ZPM model assumes an infinite basis of excited states of harmonic vibrations, and clearly this is only an approximation (and therefore a limitation) for the complicate problem of the collision between two nuclei.

We also have calculated cross sections considering couplings only to the $2^{+}$and $3^{-}$states, but in another approach within the present context by assuming Eqs. (16) and (17). Clearly these results are very similar to those obtained with the FRESCO code (see Fig. 21). In the same figure, one can observe the difference between the procedures of coupling only the $2^{+}$and $3^{-}$states in comparison with the couplings to the complete bands that correspond to these states. The difference would be much more significant if the excitation energies were smaller, because in this case several states would contribute significantly to the fusion process.

\section{DISCUSSION}

In Fig. 23, we present a summary of the results presented in this paper, by calculating the ratio between fusion data and corresponding BPM or ZPM cross sections. The exceptions are the ${ }^{84} \mathrm{Kr}+{ }^{104} \mathrm{Ru}$ and ${ }^{90} \mathrm{Zr}+{ }^{90,96} \mathrm{Zr}$ systems for which the data do not correspond to the total fusion even at subbarrier energies. At extreme sub-barrier energies, the data are underestimated by the BPM calculations by eleven orders of magnitude (see Fig. 23). On the other hand, almost all ZPM cross sections agree with the data within only two orders of magnitude (see Fig. 23). Therefore, it is reasonable to state that the couplings to inelastic excited states are responsible by the major part of the enhancements of the data relative to the BPM. Besides the data set presented in this paper, we have also analyzed data from about another 30 different systems. The corresponding results are very similar to those presented here, i.e.. the ZPM model predictions agree with most of the data within two orders of magnitude.
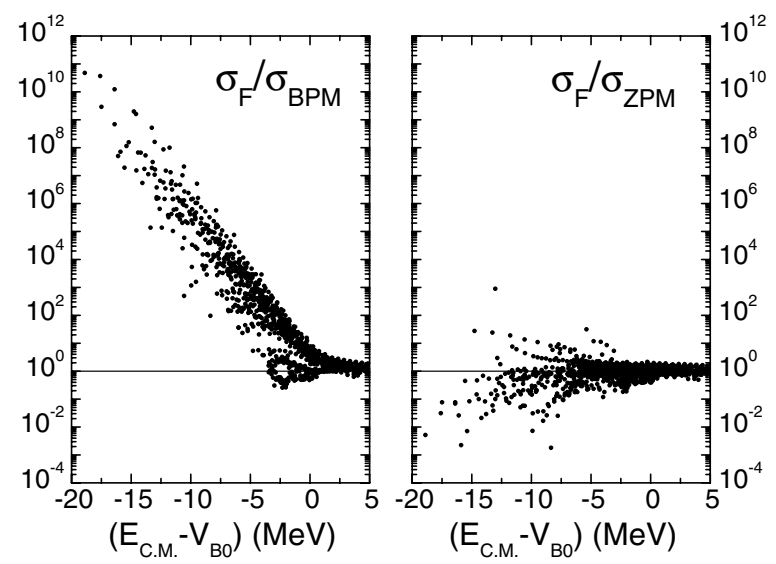

FIG. 23. Ratio between fusion data and BPM (left side) or ZPM (right side) theoretical cross sections for the systems presented from Figs. 3 to 21 . 


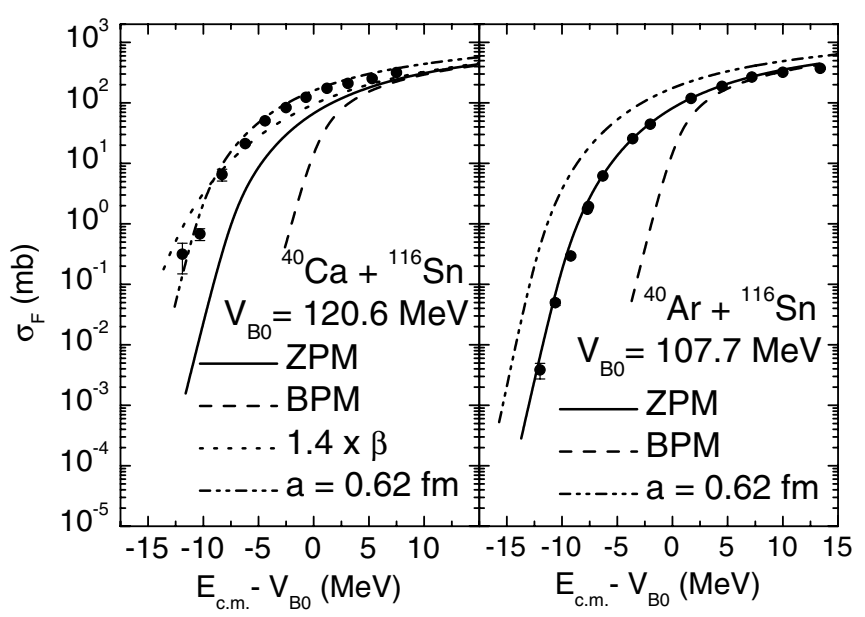

FIG. 24. Fusion cross section data (from Refs. [72,82]) for the ${ }^{40} \mathrm{Ca},{ }^{40} \mathrm{Ar}+{ }^{116} \mathrm{Sn}$ systems. The solid and dashed lines correspond to BPM and (standard) ZPM results, respectively. The other lines correspond to ZPM calculations, where the $\beta$ values or the diffuseness of the densities were modified (see text for details).

As commented in Sec. II, although some nuclei involved in our analyses are better represented by the rotational model, we have assumed the ZPM to calculate fusion cross sections. Even so, similar agreement concerning data and theoretical predictions were obtained for all systems. For instance, the ${ }^{144} \mathrm{Sm}$ is considered a vibrator while the ${ }^{154} \mathrm{Sm}$ should be better represented as a rotor. Even so, the results obtained for ${ }^{16} \mathrm{O},{ }^{40} \mathrm{Ar}+{ }^{154} \mathrm{Sm}$ (Figs. 6 and 13) are as good as those for ${ }^{16} \mathrm{O},{ }^{28} \mathrm{Si},{ }^{40} \mathrm{Ar}+{ }^{144} \mathrm{Sm}$ (Figs. 6, 7, and 14). Further examples are the ${ }^{238} \mathrm{U}$ (rotational) and ${ }^{58,64} \mathrm{Ni}$ (vibrational) nuclei, for which the corresponding results obtained for ${ }^{16} \mathrm{O}+{ }^{238} \mathrm{U}$ (Fig. 19) and ${ }^{58,64} \mathrm{Ni}+{ }^{58,64} \mathrm{Ni}$ (Figs. 17 and 21) are very similar. In this sense, the ZPM model seems to be appropriated to describe the fusion also for systems involving rotational nuclei. As already commented, probably this feature is due to the fact that Eq. (35) provides the exact result for standard deviations of both vibrational and rotational models.

In Fig. 24, we present fusion data for the ${ }^{40} \mathrm{Ca},{ }^{40} \mathrm{Ar}+{ }^{116} \mathrm{Sn}$ systems. The BPM and ZPM results correspond to the dashed and solid lines in the figure, respectively. While for the ${ }^{40} \mathrm{Ar}$ projectile the ZPM cross sections are in quite good agreement with the data, for ${ }^{40} \mathrm{Ca}$ the sub-barrier data are about three orders of magnitude larger than the ZPM predictions. This is a surprising result since both systems are in fact quite similar (same target and also same mass of the projectiles). We have investigate, as follows, whether this difference could be reduced by introducing modifications in some parameters involved in the theoretical calculations.

As commented earlier, the nuclear and Coulomb deformations should present similar, but not necessarily the same values as assumed in the ZPM calculations. On the other hand, the $\beta$ values were obtained from the transition amplitudes [Eq. (27)], which are those from Refs. $[39,40]$. The experimental transition amplitudes have, of course, experimental uncertainties. For instance, the $B(E 2)$ experimental datum for ${ }^{40} \mathrm{Ca}$ has uncertainty of about $16 \%$ [39] that results in an uncertainy of about $8 \%$ in the corresponding $\beta_{2}$ value.
Therefore, there is room for small modifications in the $\beta$ values of the ZPM calculations. To fit the data for ${ }^{40} \mathrm{Ca}+{ }^{116} \mathrm{Sn}$ we have included in our analyses an adjustable factor (the same for target and projectile) that renormalizes the $\beta$ values presented in Table I. The dotted lines in Fig. 24 represent the corresponding results. A much better agreement between data and theoretical cross sections is now obtained. However, a large and probably unrealistic factor of 1.4 is necessary to renormalize the $\beta$ values. Evidently, this difference would be even more significant if the factor were applied only relative to the $\beta$ values of ${ }^{40} \mathrm{Ca}$ (the results for ${ }^{40} \mathrm{Ar}+{ }^{116} \mathrm{Sn}$ indicates that the $\beta$ values for ${ }^{116} \mathrm{Sn}$ should not be renormalized).

Another possibility to explain the difference between the behavior of the ${ }^{40} \mathrm{Ar}+{ }^{116} \mathrm{Sn}$ and ${ }^{40} \mathrm{Ca}+{ }^{116} \mathrm{Sn}$ is related to the bare SP potential. In the present work, we have assumed the SP potential in the context of the systematics for the matter densities. This systematics has been based [19] on theoretical calculations with the Dirac-Hartree-Bogoliubov model and also on experimental results for charge distributions. As assumed here, within the systematics for the densities the radii of the $2 \mathrm{pF}$ distributions are well represented by Eq. (25), with a diffuseness value of $a=0.56 \mathrm{fm}$. However, these radius and diffuseness values represent an average behavior obtained considering the results for a large number of nuclei. Of course, small deviations around these average values are expected due to the effects of the structure of the nuclei. In Ref. [19], these variations were analyzed and standard deviations of $\sigma_{R}=$ $0.07 \mathrm{fm}$ and $\sigma_{a}=0.025 \mathrm{fm}$, relative to the average radius and diffuseness values, respectively, were found. So, it is possible to treat $R_{0}$ and $a$ as adjustable parameters. In Ref. [19] we have demonstrated that the barrier height is more affected by variations in the diffuseness value than in the radius. Thus, for ${ }^{40} \mathrm{Ca}+{ }^{116} \mathrm{Sn}$ we have assumed the diffuseness of the densities (same value for projectile and target) as an adjustable parameter to fit the data. The corresponding result is shown as dash-dotted lines in Fig. 24. A very good description of the data is now obtained, with $a=0.62 \mathrm{fm}$. At first thought, this diffuseness seems to be reasonable. Nevertheless, this value differs from the average diffuseness $(a=0.56 \mathrm{fm})$ by more than two standard deviations $\sigma_{a}=0.025 \mathrm{fm}$. This difference is even more significant because it was applied to both projectile and target nuclei. This apparently small modification of the diffuseness value from $0.56 \mathrm{fm}$ to $0.62 \mathrm{fm}$ represents, in fact, a large variation of about $50 \%$ in the strength of the undeformed nuclear potential at the s-wave barrier radius. In Fig. 25 we present theoretical Dirac-Hartree-Bogoliubov results for the matter densities of the ${ }^{40} \mathrm{Ar},{ }^{40} \mathrm{Ca}$, and ${ }^{116} \mathrm{Sn}$ nuclei (see symbols). In the same figure, $2 \mathrm{pF}$ distributions with different diffuseness values are also shown by solid and dashed lines. As expected, the average diffuseness value of the systematics [19], $a=0.56 \mathrm{fm}$, represents a much better result for the densities of the three nuclei than $a=0.62 \mathrm{fm}$. Furthermore, the theoretical density of the ${ }^{40} \mathrm{Ca}$ nucleus is very similar to that for ${ }^{40} \mathrm{Ar}$. However, if we consider the same $a=0.62 \mathrm{fm}$, obtained from fusion data fit for ${ }^{40} \mathrm{Ca}$, also for the ${ }^{40} \mathrm{Ar}+{ }^{116} \mathrm{Sn}$ system, it results in a large disagreement between fusion data and ZPM calculations (see Fig. 24). We point out that the fusion data for ${ }^{40} \mathrm{Ca}+{ }^{116} \mathrm{Sn}$ could be well fitted by considering variations in the radii instead of the diffuseness 


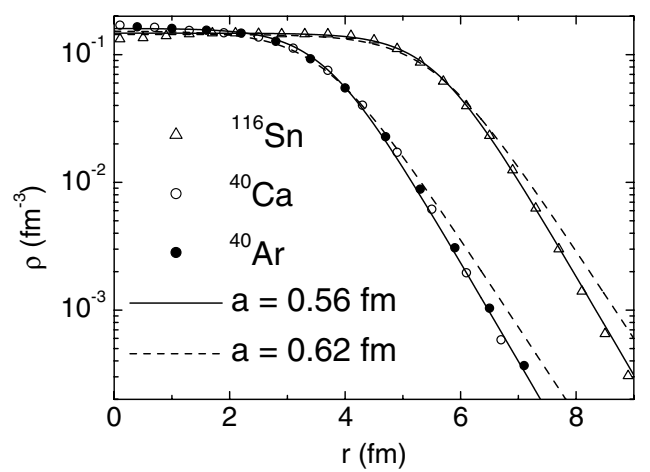

FIG. 25. The symbols represent theoretical matter densities calculated through the Dirac-Hartree-Bogoliubov model. The solid and dashed lines correspond to $2 \mathrm{pF}$ distributions with different diffuseness values. The radius of the $2 \mathrm{pF}$ distributions were obtained from Eq. (25).

of the densities. In this case, we found a variation of $\Delta R_{0} \approx$ $0.24 \mathrm{fm}$, which is also unrealistic since the standard deviation for the nuclear radii is only $\sigma_{R}=0.07 \mathrm{fm}$.

Obviously, much better agreement between fusion data and ZPM calculations could be obtained for all systems presented in this work if we had assumed $a$ and/or $\beta$ as adjustable parameters. However, this procedure is appropriate only when the resulting parameter values are within certain acceptable regions. This is a very important subject since in many works the bare potential contains adjustable parameters. In this work, we have demonstrated that an apparently small variation of $0.06 \mathrm{fm}$ in the diffuseness value (or $0.24 \mathrm{fm}$ in the radius) is in fact not realistic and may hide other possible important characteristics of the system. We point out that such a conclusion is much easier to obtain if the data analysis is performed without using any adjustable parameter. The discrepancies between the similar ${ }^{40} \mathrm{Ar}+{ }^{116} \mathrm{Sn}$ and ${ }^{40} \mathrm{Ca}+{ }^{116} \mathrm{Sn}$ systems are probably related to some effect of structure. For instance, the couplings to transfer channels may result in different contributions to the fusion process.

We turn now to the problem of extreme sub-barrier energies that is discussed in many recent works (e.g., [6-10]). In some works, the concept of extreme sub-barrier energies has been related to very small fusion cross sections. For instance, in the case of ${ }^{64} \mathrm{Ni}+{ }^{64} \mathrm{Ni}$ [8] the experimental datum at the lowest energy (at $E \approx V_{B 0}-10 \mathrm{MeV}$-see Fig. 21) corresponds to a cross section value about $10^{-5} \mathrm{mb}$. However, several systems studied here have cross sections measured at energies even lower (relative to the barrier height), with cross section values significantly larger. For example, at $E \approx V_{B 0}-$ 17.5 MeV, in the case of ${ }^{40} \mathrm{Ca}+{ }^{192}$ Os (Fig. 11), one can observe a measured cross section about $10^{-1} \mathrm{mb}$. Clearly, in this case the cross section is much larger than that for ${ }^{64} \mathrm{Ni}+{ }^{64} \mathrm{Ni}$ because the corresponding enhancement due to inelastic couplings is larger. Thus, we relate the term "extreme sub-barrier energy" to low values of energy relative to the barrier height instead of small cross sections.

In some works, it has been observed that the diffuseness values for potentials adjusted to reproduce fusion data are significantly larger than those usually assumed for elastic scattering data analyses. The nuclear interaction assumed in the present ZPM calculations has also been successfully applied to the elastic scattering process. It has also been reported that the fusion data at extreme sub-barrier energies have not been well described by $\mathrm{CC}$ calculations. For instance, in the case of ${ }^{64} \mathrm{Ni}+{ }^{64} \mathrm{Ni}$ detailed $\mathrm{CC}$ calculations were performed using adjustable parameters in the bare potential to fit the higher energy fusion data [8]. However, at energies far below the barrier the data exhibit a much steeper falloff in comparison with those theoretical results. The present ZPM calculations support this finding (see Fig. 21).

As already commented, for most of the systems studied here the ZPM predictions reproduce the data within two orders of magnitude. In Fig. 24 one can observe larger enhancements relative to BPM for lower energies (left panel). Since these enhancements arise from the effects of the couplings, a larger dispersion of the data relative to the ZPM cross sections (right panel) is expected for lower energies. However, no brusque change seems to be detected in the average behavior of the fusion cross section when approaching extreme sub-barrier energies. Even so, a more quantitative calculation may change this picture. To investigate this point, we have calculated average values for the order of magnitude of the deviations between fusion data and ZPM cross sections. We have divided the data presented in Fig. 24 (right panel) in consecutive bins of $2 \mathrm{MeV}$. For each bin we have calculated the average value of $\log \left(\sigma_{\mathrm{F}} / \sigma_{Z P M}\right)$, and we have defined average values for the order of magnitude through the following expression $\left\langle\sigma_{\mathrm{F}} / \sigma_{Z P M}\right\rangle=$ $e^{\left\langle\log \left(\sigma_{\mathrm{F}} / \sigma_{Z P M}\right)\right\rangle}$. The corresponding results are presented in Fig. 26. From above barrier energies to $5 \mathrm{MeV}$ below the barrier, in average the ZPM predictions agree with the data within $10 \%$ precision (see Fig. 26). For $-15 \leqslant E-V_{B 0} \leqslant$ $-8 \mathrm{MeV}$, in average the ZPM slightly overestimates the measured cross section by a factor about two. At extreme sub-barrier energies, the data are almost about two orders of magnitude smaller that the theoretical predictions. Evidently, these results for average values should be considered in a restricted sense because they refer only to the particular fusion data set analyzed here.

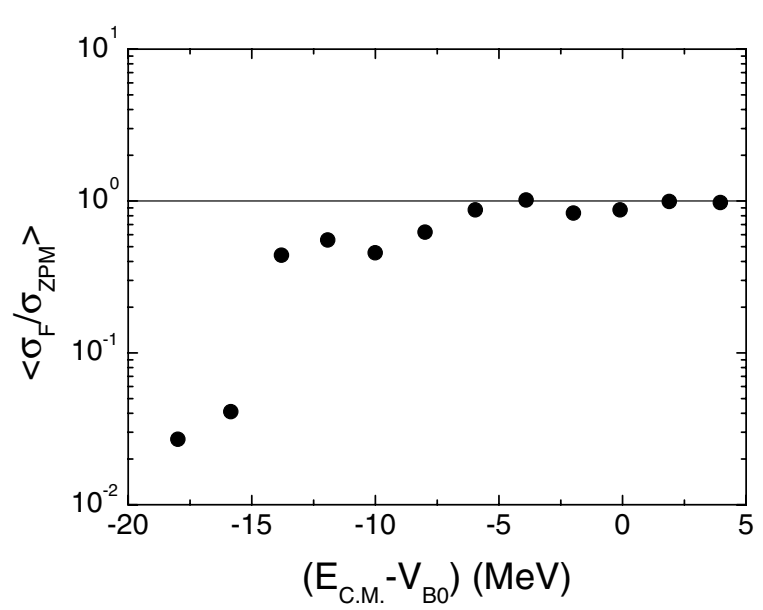

FIG. 26. Average values of the order of magnitude of the deviations between fusion data and ZPM cross sections (see text for details). 


\section{SUMMARY AND CONCLUSION}

In our theoretical calculations, we have assumed the SP potential that has been successful in describing peripheral channels for several systems in a wide energy range. Thus, the nuclear interaction used here to describe the fusion is also compatible with the heavy-ion elastic scattering process. The ZPM model takes into account the effect of the excitation energy of harmonic vibrational modes in the calculation of transmission coefficients. The model represents an approximate solution for the problem of a large number of coupled inelastic states. At very low energies the usual procedure of $\mathrm{CC}$ codes of solving a large number of $\mathrm{CC}$ equations may present numerical problems, while the ZPM results are quite accurate. Thus, the ZPM model is quite appropriate to solve problems where a large number of inelastic states present significant contribution to the fusion process, due to corresponding small excitation energies and large coupling strengths. The tunneling process strongly depends on the barrier height, which is related to variations of the distance between the surfaces of the nuclei. Thus, heavy-ion fusion is quite sensitive to vibrations of the nuclear densities. We have used the SP potential in the context of the systematics for the nuclear densities. Therefore, no free parameters were assumed in our calculations. We have analyzed fusion data for 64 heavy-ion systems involving even-even nuclei. Obviously, there are many other systems for which fusion data have been measured, but the present data set is clearly quite representative. Taking into account the lack of adjustable parameters, the overall agreement between fusion data and theoretical ZPM predictions obtained here can be considered quite satisfactory, within only two orders of magnitude for almost all the systems, including the region of extreme subbarrier energies. Therefore, the large enhancements of the data relative to undeformed BPM calculations, that reach eleven orders of magnitude at very low energies, are mostly due to the effect of the couplings to inelastic states. The remaining differences between data and ZPM cross sections can be related to several points: (i) couplings to transfer channels that are not included in our calculations, (ii) the assumption of the vibrational model for all nuclei, (iii) the assumption that $\beta_{N}=\beta_{C}$, etc. Furthermore, part of these differences could also be associated to the effect on the bare potential of variations around the corresponding adopted average values of the parameters assumed for the nuclear densities. Therefore, there is room for variations in the bare potential to obtain a better agreement between data and theoretical cross sections. In fact, we have demonstrated that significant improvement of the data description can be obtained if adjustable parameters related to coupling amplitudes and/or nuclear densities are assumed to fit the data. However, this procedure is justified only when the resulting values for these parameters are within certain acceptable regions. Indeed, we showed that even an apparently small variation of the nuclear diffuseness could be unrealistic and could hide a different characteristic of a particular system. This is a very important subject since in many works the bare interaction is related to adjustable parameters. Of course, several approximations have been assumed in our model, such as assumption of an infinity basis of harmonic oscillator states, neglecting the fact that the excitation of each surface mode corresponds to a finite transfer of angular momentum, assuming no effect of the deformation on the barrier curvature of the effective potential, and so on. Obviously, very complicated problems, such as the present question of heavy-ion collisions, always demands some degree of simplification. Therefore, if no adjustable parameters are included in the analyses, one can not expect a perfect agreement between theoretical predictions and data. In the context of averages performed over the complete data set, the ZPM model describes the fusion data from the barrier to energies as low as $15 \mathrm{MeV}$ below the barrier within a small factor of about two, but overestimates the fusion process at extreme sub-barrier energies by about two orders of magnitude. We presented the advantages and limitations of the ZPM model as a tool to predict heavy-ion fusion cross sections. The ZPM calculations have been performed using the computational code SPZPM. Optical model calculations of elastic scattering cross sections using the SP potential can be performed by the SPOMC code. Both codes are available under request to L. C. Chamon.

\section{ACKNOWLEDGMENTS}

This work was partially supported by Financiadora de Estudos e Projetos (FINEP), Fundação de Amparo à Pesquisa do Estado de São Paulo (FAPESP), and Conselho Nacional de Desenvolvimento Científico e Tecnológico (CNPq).
[1] A. B. Balantekin and N. Takigawa, Rev. Mod. Phys. 70, 77 (1998).

[2] L. C. Vaz and J. M. Alexander, Phys. Rev. C 18, 2152 (1978).

[3] R. Vandenbosch, Annu. Rev. Nucl. Sci. 42, 447 (1992).

[4] A. M. Stefanini et al., Phys. Rev. Lett. 74, 864 (1995).

[5] N. Rowley, G. R. Satchler, and P. H. Stelson, Phys. Lett. B314, 179 (1991).

[6] A. M. Stefanini et al., Phys. Rev. C 73, 034606 (2006).

[7] J. O. Newton, R. D. Butt, M. Dasgupta, D. J. Hinde, I. I. Gontchar, C. R. Morton, and K. Hagino, Phys. Lett. B586, 219 (2004).

[8] C. L. Jiang et al., Phys. Rev. Lett. 93, 012701 (2004).

[9] C. L. Jiang et al., Phys. Rev. Lett. 89, 052701 (2002).
[10] K. Hagino, N. Rowley, and M. Dasgupta, Phys. Rev. C 67, 054603 (2003).

[11] H. Esbensen, Phys. Rev. C 72, 054607 (2005).

[12] H. Esbensen, Nucl. Phys. A352, 147 (1981).

[13] H. Esbensen, Jian-Qun Wu, and G. F. Bertsch, Nucl. Phys. A411, 275 (1983).

[14] C. H. Dasso, S. Landowne, and A. Winther, Nucl. Phys. A407, 221 (1983).

[15] N. Takigawa and G. F. Bertsch, Phys. Rev. C 29, 2358 (1984).

[16] K. Hagino, N. Takigawa, J. R. Bennett, and D. M. Brink, Phys. Rev. C 51, 3190 (1995).

[17] M. A. Cândido Ribeiro, L. C. Chamon, D. Pereira, M. S. Hussein, and D. Galetti, Phys. Rev. Lett. 78, 3270 (1997). 
[18] L. C. Chamon, D. Pereira, M. S. Hussein, M. A. Cândido Ribeiro, and D. Galetti, Phys. Rev. Lett. 79, 5218 (1997).

[19] L. C. Chamon, B. V. Carlson, L. R. Gasques, D. Pereira, C. De Conti, M. A. G. Alvarez, M. S. Hussein, M. A. Cândido Ribeiro, E. S. Rossi Jr., and C. P. Silva, Phys. Rev. C 66, 014610 (2002).

[20] J. J. S. Alves et al., Nucl. Phys. A748, 59 (2005).

[21] D. Pereira, C. P. Silva, J. Lubian, E. S. Rossi Jr., and L. C. Chamon, Phys. Rev. C 73, 014601 (2006).

[22] L. C. Chamon, D. Pereira, and M. S. Hussein, Phys. Rev. C 58, 576 (1998).

[23] M. A. G. Alvarez, L. C. Chamon, D. Pereira, E. S. Rossi Jr., C. P. Silva, L. R. Gasques, H. Dias, and M. O. Roos, Nucl. Phys. A656, 187 (1999).

[24] L. R. Gasques, L. C. Chamon, C. P. Silva, D. Pereira, M. A. G. Alvarez, E. S. Rossi Jr., V. P. Likhachev, B. V. Carlson, and C. De Conti, Phys. Rev. C 65, 044314 (2002).

[25] E. S. Rossi Jr., D. Pereira, L. C. Chamon, C. P. Silva, M. A. G. Alvarez, L. R. Gasques, J. Lubian, B. V. Carlson, and C. De Conti, Nucl. Phys. A707, 325 (2002).

[26] L. R. Gasques et al., Phys. Rev. C 67, 024602 (2003).

[27] T. Tarutina, L. C. Chamon, and M. S. Hussein, Phys. Rev. C 67, 044605 (2003).

[28] M. A. G. Alvarez, L. C. Chamon, M. S. Hussein, D. Pereira, L. R. Gasques, E. S. Rossi Jr., and C. P. Silva, Nucl. Phys. A723, 93 (2003).

[29] L. R. Gasques, L. C. Chamon, D. Pereira, M. A. G. Alvarez, E. S. Rossi Jr., C. P. Silva, G. P. A. Nobre, and B. V. Carlson, Phys. Rev. C 67, 067603 (2003).

[30] P. R. S. Gomes et al., Phys. Rev. C 70, 054605 (2004).

[31] M. A. G. Alvarez, N. Alamanos, L. C. Chamon, and M. S. Hussein, Nucl. Phys. A753, 83 (2005).

[32] P. R. S. Gomes et al., Phys. Rev. C 71, 034608 (2005).

[33] J. J. S. Alves et al., Braz. J. Phys. 35, 909 (2005).

[34] P. R. S. Gomes et al., J. Phys. G 31, S1669 (2005).

[35] P. R. S. Gomes et al., Phys. Lett. B634, 356 (2006).

[36] D. L. Hill and J. A. Wheeler, Phys. Rev. 89, 1102 (1953).

[37] L. C. Chamon, G. P. A. Nobre, D. Pereira, E. S. Rossi Jr., C. P. Silva, L. R. Gasques, and B. V. Carlson, Phys. Rev. C 70, 014604 (2004).

[38] B. V. Carlson, L. C. Chamon, and L. R. Gasques, Phys. Rev. C 70, 057602 (2004).

[39] S. Raman, C. H. Malarkey, W. T. Milner, C. W. Nestor Jr., and P. H. Stelson, At. Data Nucl. Data Tables 36, 1 (1987).

[40] T. Kibedi and R. H. Spear, At. Data Nucl. Data Tables 80, 35 (2002).

[41] H. Esbensen and S. Landowne, Phys. Rev. C 35, 2090 (1987).

[42] C. H. Dasso and S. Landowne, Z. Phys. A 322, 175 (1985).

[43] I. J. Thompson, Comput. Phys. Rep. 7, 167 (1988).

[44] M. G. Mazarakis and W. E. Stephens, Phys. Rev. C 7, 1280 (1973).

[45] M. D. High and B. Cujec, Nucl. Phys. A282, 181 (1977).

[46] J. R. Patterson, H. Winkler, and C. S. Zaidnis, Astrophys. J. 157, 367 (1969).

[47] D. G. Kovar et al., Phys. Rev. C 20, 1305 (1979).

[48] R. Rosales et al., Rev. Mex. Fis. 49, 88 (2003).

[49] J. Thomas, Y. T. Chen, S. Hinds, D. Meredith, and M. Olson, Phys. Rev. C 33, 1679 (1986).

[50] G. Hulke, C. Rolfs, and H. P. Trautvetter, Z. Phys. A 297, 161 (1980).

[51] A. Kuronen, J. Keinonen, and P. Tikkanen, Phys. Rev. C 35, 591 (1986)
[52] S. C. Wu and C. A. Barnes, Nucl. Phys. A422, 373 (1983).

[53] J. O. Newton, C. R. Morton, M. Dasgupta, J. R. Leigh, J. C. Mein, D. J. Hinde, H. Timmers, and K. Hagino, Phys. Rev. C 64, 064608 (2001).

[54] S. Santra, P. Singh, S. Kailas, A. Chatterjee, A. Shrivastava, and K. Mahata, Phys. Rev. C 64, 024602 (2001).

[55] E. Vulgaris, L. Grodzins, S. G. Steadman, and R. Ledoux, Phys. Rev. C 33, 2017 (1986).

[56] H. Timmers, Ph.D. thesis, The Australian National University, 1996.

[57] J. R. Leigh et al., Phys. Rev. C 52, 3151 (1995).

[58] C. R. Morton, A. C. Berriman, M. Dasgupta, D. J. Hinde, J. O. Newton, K. Hagino, and I. J. Thompson, Phys. Rev. C 60, 044608 (1999).

[59] S. Mitsuoka, H. Ikezoe, K. Nishio, and J. Lu, Phys. Rev. C 62, 054603 (2000).

[60] D. J. Hinde, W. Pan, A. C. Berriman, R. D. Butt, M. Dasgupta, C. R. Morton, and J. O. Newton, Phys. Rev. C 62, 024615 (2000).

[61] R. G. Stokstad, Y. Eisen, S. Kaplanis, D. Pelte, U. Smilansky, and I. Tserruya, Phys. Rev. C 21, 2427 (1980).

[62] M. Dasgupta, A. Navin, Y. K. Agarwal, C. V. K. Baba, H. C. Jain, M. L. Jhingan, and A. Roy, Nucl. Phys. A539, 351 (1992).

[63] D. Ackermann et al., Nucl. Phys. A609, 91 (1996).

[64] M. Dasgupta et al., Proceedings of the Workshop on Heavy-ion Fusion: Exploring the Variety of Nuclear Proprieties, Padova, Italy, 1994, (World Scientific Press, Singapore, 1994), p. 115.

[65] R. Pengo, D. Evers, K. E. G. Löbner, U. Quade, K. Rudolph, S. J. Skorka, and I. Weidl, Nucl. Phys. A411, 255 (1983).

[66] A. M. Stefanini, L. Corradi, A. M. Vinodkumar, Yang Feng, F. Scarlassara, G. Montagnoli, S. Beghini, and M. Bisogno, Phys. Rev. C 62, 014601 (2000).

[67] H. A. Aljuwair, R. J. Ledoux, M. Beckerman, S. B. Gazes, J. Wiggins, E. R. Cosman, R. R. Betts, S. Saini, and Ole Hansen, Phys. Rev. C 30, 1223 (1984).

[68] M. Beckerman, M. Salomaa, A. Sperduto, J. D. Molitoris, and A. DiRienzo, Phys. Rev. C 25, 837 (1982).

[69] A. A. Sonzogni, J. D. Bierman, M. P. Kelly, J. P. Lestone, J. F. Liang, and R. Vandenbosch, Phys. Rev. C 57, 722 (1998).

[70] J. D. Bierman, P. Chan, J. F. Liang, M. P. Kelly, A. A. Sonzogni, and R. Vandenbosch, Phys. Rev. C 54, 3068 (1996).

[71] H. Timmers, D. Ackermann, S. Beghini, L. Corradi, J. H. He, G. Montagnoli, F. Scarlassara, A. M. Stefanini, and N. Rowley, Nucl. Phys. A633, 421 (1998).

[72] W. Reisdorf et al., Nucl. Phys. A438, 212 (1985).

[73] W. Reisdorf, F. P. Hessberger, K. D. Hildenbrand, S. Hofmann, G. Münzenberg, K. H. Schmidt, W. F. W. Schneider, K. Sümmerer, G. Wirth, J. V. Kratz, K. Schutt, and C. C. Sahm, Nucl. Phys. A444, 154 (1985).

[74] J. G. Keller, K.-H Schmidt, F. P. Hessberger, G. Munzenberg, W. Reisdorf, H.-G. Clerc, and C.-C. Sahm, Nucl. Phys. A452, 173 (1986).

[75] M. Beckerman, M. Salomaa, A. Sperduto, J. D. Molitoris, and A. Di Rienzo, Phys. Rev. C 25, 837 (1982).

[76] M. Beckerman, J. Ball, H. Enge, M. Salomaa, A. Sperduto, S. Gazes, A. Di Rienzo, and J. D. Molitoris, Phys. Rev. C 23, 1581 (1981).

[77] F. L. H. Wolfs, Phys. Rev. C 36, 1379 (1987).

[78] C. L. Jiang et al., Phys. Rev. C 71, 044613 (2005). 
[79] D. J. Hinde, M. Dasgupta, J. R. Leigh, J. C. Mein, C. R. Morton, J. O. Newton, and H. Timmers, Phys. Rev. C 53, 1290 (1996).

[80] D. J. Hinde, C. R. Morton, M. Dasgupta, J. R. Leigh, J. C. Mein, and H. Timmers, Nucl. Phys. A592, 271 (1995).
[81] A. M. Stefanini, D. Ackermann, L. Corradi, J. H. He, G. Montagnoli, S. Beghini, F. Scarlassara, and G. F. Segato, Phys. Rev. C 52, R1727 (1995).

[82] F. Scarlassara, S. Beghini, G. Montagnoli, G. F. Segato, D. Ackerman, L. Corradi, C. J. Lin, A. M. Stefanini, and L. F. Zheng, Nucl. Phys. A672, 99 (2000). 